\title{
COMPLEX ALGEBRAIC GEOMETRY AND CALCULATION OF MULTIPLICITIES FOR INDUCED REPRESENTATIONS OF NILPOTENT LIE GROUPS
}

\author{
L. CORWIN AND F. P. GREENLEAF
}

\begin{abstract}
Let $G$ be a connected, simply connected nilpotent Lie group, $H$ a Lie subgroup, and $\sigma$ an irreducible unitary representation of $H$. In a previous paper, the authors and G. Grelaud gave an explicit direct integral decomposition (with multiplicities) of $\operatorname{Ind}(H \uparrow G, \sigma)$. One consequence of that work was that the multiplicity function was either a.e. infinite or a.e. bounded. In this paper, it is proved that if the multiplicity function is bounded, its parity is a.e. constant. The proof is algebraic-geometric in nature and amounts to an extension of the familiar fact that for almost all polynomials over $R$ of fixed degree, the parity of the number of roots is a.e. constant. One consequence of the methods is that if $G$ is a complex nilpotent Lie group and $H$ a complex Lie subgroup, then the multiplicity is a.e. constant.
\end{abstract}

1. Let $K$ be a connected, closed subgroup of the connected, simply connected real nilpotent Lie group $G$, and let $\chi$ be an irreducible unitary representation of $K$. In [2], the authors and G. Grelaud gave a direct integral decomposition of the induced representation $\rho=\operatorname{Ind}(K \uparrow G, \chi)$ into irreducibles: $\rho \cong \int_{G^{\prime}}^{\oplus} m(\pi)$. $\pi d \nu(\pi)$. The spectrum, supp $\nu$, and the multiplicities, $m(\pi)$, were computed in a natural geometric way in terms of coadjoint orbits. We were also able to show, as a consequence of our methods, that

$$
\text { either } m(\pi) \equiv \infty \text { or there is a bound } N \text { with } m(\pi) \leq N
$$

(of course, this holds $\nu$-almost everywhere). In the examples computed in [2], it was also always true that when $m(\pi)$ was finite, it had constant parity (was always even or always odd) $\nu$-a.e. Roger Howe asked us whether this was always the case, and one of the main purposes of our paper is to prove that it is. Our methods also enable us to get a multiplicity result for complex nilpotent Lie groups as well. We feel that this approach may prove useful in other areas, notably the decomposition of $\left.\pi\right|_{K}$ for $\pi \in G^{\wedge}$ (a question currently under investigation).

The basic results we prove in this paper are these:

THEOREM 1. Let $\mathfrak{g}$ be a complex nilpotent Lie algebra and $\mathfrak{k}$ a complex subalgebra, and let $G, K$ be the associated simply connected Lie groups (regarded as real Lie groups). Let $\chi$ be an irreducible unitary representation of $K$, and let

Received by the editors July 15, 1986.

1980 Mathematics Subject Classification (1985 Revision). Primary 22E27; Secondary 22E45.

The first author was on sabbatical leave from Rutgers University during part of the preparation of this paper. He is grateful to Rutgers for financial support and to the Institute for Advanced Study for its hospitality. Research also supported in part by NSF Grant MCS 84-02704; second author supported by NSF Grant 85-01945. 
$\rho=\operatorname{Ind}(K \uparrow G, \chi)$. Then $\rho$ has uniform multiplicity: in the direct integral decomposition of $\rho$ into irreducibles,

$$
\rho \cong \int_{G^{\wedge}}^{\oplus} m(\pi) \cdot \pi \nu(d \pi),
$$

we have $m(\pi)=$ constant, $\nu$-a.e. (The constant may be $\infty$.)

THEOREM 2. Let $G_{0}$ be a real connected, simply connected nilpotent Lie group, $K_{0}$ a closed connected subgroup, and $\chi$ an irreducible unitary representation of $K_{0}$. Set $\rho=\operatorname{Ind}\left(K_{0} \uparrow G_{0}, \chi\right)$. Then in the direct integral decomposition of $\rho$ into irreducibles, $\rho \cong \int_{G_{0}^{\hat{0}}}^{\oplus} m(\pi) \cdot \pi \nu(d \pi)$, either

(i) $m(\pi) \equiv+\infty$ or

(ii) $m(\pi)$ is bounded and has constant parity for $\nu$-a.e. $\pi$.

The ideas behind the two proofs are similar; both use complex algebraic geometry. However, the details are quite different. In Theorem 1, we are working directly with varieties over $\mathbf{C}$, while in Theorem 2 we need to complexify the Kirillov orbit picture. Thus the two proofs are virtually independent. In both cases, we must outline the detailed concordance between the real and complex pictures for the main facts of [2], which were proved there only for real fields, and for the Pukanszky parametrization of all coadjoint orbits. Regarding [2], we shall try to state clearly the results and adaptations needed for this paper, in order to make this account self-contained.

The following is the organization of the rest of this paper: $\S 2$ contains an account of the main results of [2], and concludes with a sketch of the proofs of Theorems 1 and 2 . In $\S 3$, we give the main results we need from algebraic geometry. $\S 4$ gives some results on coadjoint orbits, needed for the proof of Theorem 1 ; we prove Theorem 1 in $\S 5$. In $\S 6$, we give results comparing coadjoint orbits for a nilpotent Lie group and its complexification; we use these in $\S 7$, where Theorem 2 is proved. $\S 8$ contains some examples.

It is a pleasure to thank David Rohrlich for his assistance with some of the results from algebraic geometry cited in this paper.

2. We shall repeatedly refer to Pukanszky's method [6] for parametrizing all the orbits in a vector space under the action of a connected unipotent Lie group; we shall also need a number of basic facts from the paper [2]. Here we assemble these results for future reference.

In what follows, we use $\mathbf{F}$ to stand for $\mathbf{R}$ or $\mathbf{C}$; in fact, for most statements $\mathbf{F}$ could be any field of characteristic 0. The theorem in [6] (see also [1]) on parametrization of orbits is given for $\mathbf{F}=\mathbf{R}$, but the proof applies verbatim when $\mathbf{F}=\mathbf{C}$.

If $V$ is a vector space over $\mathbf{F}$ and $\mathfrak{g}$ is a Lie algebra (over $\mathbf{F}$ ) acting nilpotently on $V$, we may assume that $\mathfrak{g}$ is nilpotent by factoring out the kernel. Then $G=\left\{e^{X}: X \in \mathfrak{g}\right\}$ is a simply connected nilpotent Lie group (over $\mathbf{F}$ ) acting unipotently on $V$. Let $\left\{l_{1}, \ldots, l_{n}\right\}$ be a Jordan-Hölder basis for $V$. Then $V_{j}=\mathbf{F}$ $\operatorname{span}\left\{l_{j+1}, \ldots, l_{n}\right\}$ is $G$-invariant for all $j\left(V_{n}=(0)\right.$ by definition), and we obtain quotient actions of $\mathfrak{g}$ and $G$ on $V / V_{j}$ which commute with the canonical projections $P_{j}: V \rightarrow V / V_{j}$. If $l \in V$, define

$$
e_{j}(l)=\operatorname{dim}_{\mathbf{F}}\left(G \cdot P_{j}(l)\right), \quad e(l)=\left(e_{1}(l), \ldots, e_{n}(l)\right) \in \mathbf{Z}^{n} .
$$


The stabilizer $\operatorname{Stab}_{G}(l)=\{x \in G: x \cdot l=l\}$ is a connected Lie subgroup of $G$ whose Lie algebra is $\mathfrak{p}(l)=\{X \in \mathfrak{g}: X \cdot l=0\} ; \mathfrak{p}(l)$ is an algebra over $\mathbf{F}$, and the orbit $G \cdot l$ is a complex manifold if $\mathbf{F}=\mathbf{C}$. Similar remarks hold for the quotient actions.

The result from [6] (as modified in [1]) that we need is

(2.1) THEOREM (PARAMETRIZATION OF ORBITS). Let $\mathfrak{g}$ be a nilpotent Lie algebra over $\mathbf{F}$ acting nilpotently on a $\mathbf{F}$-vector space $V$. Fix a Jordan-Hölder basis for $V$; define $G$, the $V_{j}$, the quotient actions $G \times V / V_{j} \rightarrow V / V_{j}$, and the n-tuples $e(l)$ for $l \in V$ as above. Let $\mathcal{E}=\left\{e\right.$ : there exists $l \in V$ with $e_{j}=e_{j}(l)$ for $\left.1 \leq j \leq n\right\}$; for $e \in \mathcal{E}$, define $U_{e}=\{l \in V: e(l)=e\}$. Then the $U_{e}$ partition $V$, and

(i) $e \in \mathcal{E} \Rightarrow e_{j}=e_{j-1}$ or $e_{j}=e_{j-1}+1, \forall j$ (we set $\left.e_{0}=0\right)$. In particular, $\mathcal{E}$ is finite.

(ii) There is an ordering of indices $\mathcal{E}=\left\{e^{(1)}>\cdots>e^{(r)}\right\}$ such that $(\forall e \in$ $\varepsilon) \bigcup_{e^{\prime} \geq e} U_{e^{\prime}}$ is a Zariski-open set in $V$. In particular, every $U_{e}$ is Zariski-open in the Zariski-closed set $V \sim \bigcup_{e^{\prime}>e} U_{e^{\prime}}$; furthermore, $U_{e^{(1)}}$ is Zariski-open in $V$.

(iii) Each layer $U_{e}$ is $G$-invariant, and all orbits in $U_{e}$ have the same $\mathbf{F}$-dimension.

Fix $e \in \mathcal{E}$, and define index sets $S(e)=\left\{j: e_{j}=1+e_{j-1}\right\}, T(e)=\left\{j: e_{j}=e_{j-1}\right\}$. There is a direct sum decomposition $V=V_{T(e)} \oplus V_{S(e)}$, where $V_{T(e)}=\mathbf{F}$-span $\left\{l_{j}: j \in\right.$ $T(e)\}, V_{S(e)}=\mathbf{F}-\operatorname{span}\left\{l_{j}: j \in S(e)\right\}$. Then

(iv) $\Sigma_{e}=U_{e} \cap V_{T(e)}$ is a nonempty set in $V_{T(e)}$, and is Zariski-open in the Zariski-closed set $V_{T(e)} \sim\left(\bigcup_{e^{\prime}>e} U_{e^{\prime}}\right)$.

(v) The elements of $\Sigma_{e}$ give a cross-section for the $G$-orbits in $U_{e}$, and the semialgebraic set $\Sigma=\bigcup_{e} \Sigma_{e}$ is a cross-section for all $G$-orbits in $V$.

(vi) There is an F-birational nonsingular map $Q_{e}: \Sigma_{e} \times V_{S(e)} \rightarrow U_{e}$ such that for each $l \in \Sigma_{e}, Q_{e}$ maps $\{l\} \times V_{S(e)}$ to $G \cdot l$. This map is polynomial on $V_{S(e)}$ for each $l \in \Sigma_{e}$, and the inverse $P_{e}: U_{e} \rightarrow \Sigma_{e} \times V_{S(e)}$ is given by

$$
P_{e}(l)=\left(G \cdot l \cap V_{T(e)}, \pi_{S}(l)\right),
$$

where $\pi_{S}, \pi_{T}$ are the projections identifying $V$ with $V_{S(e)} \oplus V_{T(e)}$. In particular, $\pi_{S} \circ Q_{e}: \Sigma_{e} \times V_{S(e)} \rightarrow V_{S(e)}$ is the projection on the second factor. (This shows that each orbit is the graph of a polynomial.)

The sets $U_{e}$ are called "layers" in $V$, and the collection $\left\{U_{e}\right\}$ is an "E-layering" of $V$. We write $\tilde{P}_{e}=\pi_{T} \circ P_{e}: U_{e} \rightarrow \Sigma_{e}$.

In Kirillov theory, one applies this result to the coadjoint action: a real nilpotent Lie group $G_{0}$ acts on its Lie algebra $g_{0}$ by Ad, and thus on $\mathfrak{g}_{0}^{*}=\operatorname{Hom}_{\mathbf{R}}\left(\mathfrak{g}_{0}, \mathbf{R}\right)$ by the coadjoint action $\mathrm{Ad}^{*}$. If $X_{1}, \ldots, X_{n}$ is a strong Malcev basis for $g_{0}$ (i.e., $\mathbf{R}$-span $\left\{X_{1}, \ldots, X_{j}\right\}$ is an ideal, $\left.1 \leq j \leq n\right)$, the dual basis $\left\{l_{1}, \ldots, l_{n}\right\}$ in $\mathbf{g}_{0}^{*}$ is Jordan-Hölder and determines an $\mathcal{E}$-layering of $\mathfrak{g}_{0}^{*}$.

In this paper we must consider some other situations. Let $\mathfrak{g}$ be a nilpotent Lie algebra over $\mathbf{C}$, and let $\mathfrak{g}_{\mathbf{R}}$ be $\mathfrak{g}$ with the field reduced to $\mathbf{R}$. Then $G=\exp \mathfrak{g}$ is a complex Lie group; it acts on both $\mathfrak{g}$ and $\mathfrak{g}_{\mathbf{R}}$. Thus we have two coadjoint actions of $G$ on $\mathfrak{g}^{*}=\operatorname{Hom}_{\mathbf{C}}(\mathfrak{g}, \mathbf{C})$, and on $\mathfrak{g}_{\mathbf{R}}^{*}=\operatorname{Hom}_{\mathbf{R}}\left(\mathfrak{g}_{\mathbf{R}}, \mathbf{R}\right)$. Theorem 2.1 applies to both of them. If one chooses nicely related strong Malcev bases in $\mathfrak{g}$ and $\mathfrak{g}_{\mathbf{R}}$, then the dual bases in $\mathfrak{g}^{*}, \mathfrak{g}_{\mathbf{R}}^{*}$ will be related in a way that lets us set up a concordance between the layerings in $\mathfrak{g}^{*}$ and $\mathfrak{g}_{\mathbf{R}}^{*}$. This will be done explicitly in $\S 4$, as the first step in proving Theorem 1. In proving Theorem 2, we face a different problem: 
we start with a real algebra $\mathfrak{g}_{0}$ and form its complexification $\mathfrak{g}=\mathfrak{g}_{0} \otimes \mathbf{C}$, and we must relate layerings in $\mathfrak{g}_{0}^{*}$ with those in $\mathfrak{g}^{*}=\operatorname{Hom}_{\mathbf{C}}(\mathfrak{g}, \mathbf{C})$. Here we have different groups $G_{0}, G$ ( $G$ is the group corresponding to $\mathfrak{g}$ ) acting on different spaces $\mathfrak{g}_{0}^{*}, \mathfrak{g}^{*}$. We analyze these actions in $\S 6$.

The basic result of [2] is stated in terms of the $\mathcal{E}$-layering. We recall it here because we will have to devise some variants. Let $\mathfrak{k}_{0}$ be a (real) subalgebra of the real nilpotent Lie algebra $\mathfrak{g}_{0}$; let $G_{0}=\exp \mathfrak{g}_{0}, K_{0}=\exp \mathfrak{k}_{0}$. Let $P: \mathfrak{g}_{0}^{*} \rightarrow \mathfrak{k}_{0}^{*}$ be the canonical projection, and let $\chi \in K_{0}^{\widehat{b}}$ be an irreducible unitary representation with coadjoint orbit $O_{\chi}=K_{0} \cdot f_{0} \subseteq \mathfrak{k}_{0}^{*}$ (here, $f_{0} \in \mathfrak{k}_{0}^{*}$ ). Fix a strong Malcev basis $X_{1}, \ldots, X_{n}$ in $\mathfrak{g}_{0}$, let $l_{1}, \ldots, l_{n}$ be the dual basis in $\mathfrak{g}_{0}^{*}$, and partition $\mathfrak{g}_{0}^{*}$ into layers $\left\{U_{e}: e \in \mathcal{E}\right\}$. Now let $e \in \mathcal{E}$ be the largest index such that $U_{e} \cap P^{-1}\left(O_{\chi}\right) \neq \varnothing$; this intersection is Zariski-open and dense in the (irreducible) algebraic variety $P^{-1}\left(O_{\chi}\right)$, and $\Sigma_{e}^{\chi}=\pi_{T} \circ P_{e}\left(U_{e} \cap P^{-1}\left(O_{\chi}\right)\right)$ is a semialgebraic set of representatives for the (generic) $G_{0}$-orbits in $\mathfrak{g}_{0}^{*}$ that meet $P^{-1}\left(O_{\chi}\right)$.

(2.2) THEOREM [2] (MULTIPLICITY THEOREM). Let $\mathrm{g}_{0}$ be a real nilpotent Lie algebra, $\mathfrak{k}_{0}$ a subalgebra; let $G_{0}, K_{0}$ be the corresponding connected, simply connected groups, and let $\chi \in K_{0}^{\widehat{.}}$. Defining an $\mathcal{E}$-layering for $\mathfrak{g}_{0}^{*}$ as above, let $[\nu]$ be the canonical measure class on $\Sigma_{e}^{\chi}$, and write $G_{0} \cdot l=\operatorname{Ad}^{*}\left(G_{0}\right) l$ for $l \in g_{0}^{*}$. Define the "defect index"

$$
\begin{aligned}
\tau_{0}= & \operatorname{generic}\left(\operatorname{dim}_{\mathbf{R}} G_{0} \cdot l: l \in P^{-1}\left(O_{\chi}\right)\right) \\
& -2 \operatorname{generic}\left(\operatorname{dim}_{\mathbf{R}} K_{0} \cdot l: l \in P^{-1}\left(O_{\chi}\right)\right)+\operatorname{dim}_{\mathbf{R}} O_{\chi} .
\end{aligned}
$$

Then if $\tau_{0}>0$, we have

$$
\rho=\operatorname{Ind}\left(K_{0} \uparrow G_{0}, \chi\right) \cong \int_{\Sigma_{e}^{\chi}} \infty \cdot \pi_{l} d \nu(l) .
$$

If $\tau_{0}=0$, then for $\nu$-a.e. $l \in \Sigma_{e}^{\chi}$, we have

(i) $G_{0} \cdot l \cap P^{-1}\left(O_{\chi}\right)$ is a closed submanifold.

(ii) There is a uniform bound for

$$
m(l)=\text { number of connected components in } G_{0} \cdot l \cap P^{-1}\left(O_{\chi}\right) .
$$

(iii) $m(l)=$ number of $\operatorname{Ad}^{*}\left(K_{0}\right)$-orbits in $G_{0} \cdot l \cap P^{-1}\left(O_{\chi}\right)$, and

$$
\rho \cong \int_{\Sigma_{e}^{x}}^{\oplus} m(l) \pi_{l} d \nu(l) .
$$

Note. We will recall the definitions of semialgebraic sets and their canonical measure classes in the next section.

Since $\chi$ is monomial, it will always be possible for us to assume, by changing $K_{0}$, that $\chi$ is 1 -dimensional. (In the case of Theorem 1 , we will have to show that every irreducible representation of a complex group is induced from a 1-dimensional representation of a complex subgroup-see §4.) Thus we shall assume from now on that $\operatorname{dim} \chi=1$. Then

$$
\chi(y)=e^{2 \pi i f_{0}(\log y)}
$$

where $f_{0} \in \mathfrak{k}_{0}^{*}$ is a Lie homomorphism. Thus $O_{\chi}=K_{0} \cdot f_{0}$ is a single point and $P^{-1}\left(O_{\chi}\right)$ is flat. In proving Theorems 1 and 2 , we will make use of a subsidiary result used in [2] for proving Theorem 2.2. 
(2.5) Proposition. With notation as in Theorem 2.2, assume that $\operatorname{dim} \chi=1$; define $f_{0}$ as in (2.4), and let $f^{\prime} \in P^{-1}\left(f_{0}\right)$. Then there is a subspace $E_{1}^{0}$ of $\mathfrak{k}_{0}^{\perp}$ such that

$$
\rho \cong \int_{f^{\prime}+E_{1}^{0}}^{\oplus} \pi_{l} d \mu(l)
$$

where $d \mu(l)$ is Lebesgue measure.

We can now explain the idea behind the proof of Theorem 2. The formula (2.6) makes it plausible that the number $m(l)$ of Theorem 2.2 is equal (a.e.) to $\operatorname{Card}(G \cdot l) \cap\left(f^{\prime}+E_{1}^{0}\right)$; we shall prove this (modulo results in [2]) in §3. (We may assume that $\tau_{0}=0$, since otherwise $m(l) \equiv \infty$.) We thus wish to know. that $\operatorname{Card}(G \cdot l) \cap\left(f^{\prime}+E_{1}^{0}\right)$ has constant parity. To prove this, complexify everything: let $\mathfrak{g}=\mathfrak{g}_{0} \otimes_{\mathbf{R}} \mathbf{C}, \mathfrak{k}=\mathfrak{k}_{0} \otimes_{\mathbf{R}} \mathbf{C}$, and let $G, K$ be the corresponding Lie groups. Similarly, let $\mathfrak{g}^{*}=\mathfrak{g}_{0}^{*} \otimes_{\mathbf{R}} \mathbf{C}=\mathfrak{g}_{0}^{*}+i \mathfrak{g}_{0}^{*}, \mathfrak{k}^{*}=\mathfrak{k}_{0}^{*} \otimes_{\mathbf{R}} \mathbf{C}=\mathfrak{k}_{0}^{*}+i \mathfrak{k}_{0}^{*}$. In $\mathfrak{g}^{*}$ we consider $f^{\prime}+\mathbf{C} E_{1}^{0}$, the Zariski-closure of $f^{\prime}+E_{1}^{0} \subseteq \mathfrak{g}_{0}^{*}$. Generically, orbits $G \cdot l$ for the action of $G$ on $l \in \mathfrak{g}^{*}$ must meet $f^{\prime}+\mathbf{C} E_{1}^{0}$ in isolated points, just as orbits $G_{0} \cdot l$ meet $f^{\prime}+E_{1}^{0}$ discretely if $l \in \mathfrak{g}_{0}^{*}$. Let $\left(\Sigma_{e}^{\chi}\right)^{\mathbf{C}}$ be the Zariski-closure in $\mathfrak{g}^{*}$ of $\Sigma_{e}^{\chi} \subseteq \mathfrak{g}_{0}^{*}$. The Pukanszky parametrization of orbits applies to both the action of $G$ on $\mathfrak{g}^{*}$ and the action of $G_{0}$ on $\mathfrak{g}_{0}^{*}$, and there is a natural concordance between the two situations. In the complex situation we obtain a map taking a point $l \in f^{\prime}+\mathbf{C} E_{1}^{0}$ to its orbit representative, which lies in $\left(\Sigma_{e}^{\chi}\right)^{\mathrm{C}}$. This map is rational and nonsingular, and it is finite-to-one on a Zariski-open set. With a little effort it can be regarded as a polynomial map from $f^{\prime}+\mathbf{C} E_{1}^{0}$ to $\left(\Sigma_{e}^{\chi}\right)^{\mathbf{C}}$. It can be shown that both varieties are irreducible and have the same dimension over $\mathbf{C}$. By a result in algebraic geometry, we can remove negligible sets from each variety and obtain a covering map (in the Euclidean topology). Thus

$$
n(l)=\operatorname{Card}\left[G \cdot l \cap\left(f^{\prime}+\mathbf{C} E_{1}^{0}\right)\right]
$$

is defined and constant on a topologically connected Zariski-open set in $\left(\Sigma_{e}^{\chi}\right)^{\mathrm{C}}$.

Now suppose that $l$ is real: $l \in\left(\Sigma_{e}^{\chi}\right)^{\mathbf{C}} \cap\left(\mathfrak{g}_{0}^{*}+i 0\right)$. The nonreal points in $G \cdot l \cap\left(f^{\prime}+\mathbf{C} E_{1}^{0}\right)$ come in conjugate pairs, and hence the parity of $n_{0}(l)=$ $\operatorname{Card}\left[G \cdot l \cap\left(f^{\prime}+E_{1}^{0}\right)\right]$ is constant on a Zariski-open set in $\Sigma_{e}^{\chi}$. It is not hard to show that for real $l, G \cdot l \cap\left(\mathfrak{g}_{0}^{*}\right)=G_{0} \cdot l$. Thus $n_{0}(l)$ has essentially constant parity. Finally, one observes that $n_{0}(l)=m(l)$-except for a few measure-theoretic details, this is Proposition 2.5- to complete the proof.

Theorem 1 is similar but easier. The main observation is that in this setting, $\mathfrak{g}_{\mathbf{R}}^{*}$ and $\mathfrak{k}_{\mathbf{R}}^{*}$ can be regarded as complex vector spaces, and $E_{1}$ is a complex subspace. Thus the map of $f^{\prime}+\mathbf{C} E_{1}$ to $\Sigma_{e}^{\chi}$ obtained by restricting $\tilde{P}$ is essentially a covering map, and hence $n(l)=\operatorname{Card}\left(G \cdot l \cap\left(f^{\prime}+E_{1}\right)\right)$ is essentially constant. One then shows (via Proposition 2.5) that $n(l)=m(l)$ to complete the proof.

3. Here we give some facts from algebraic geometry that will be used repeatedly. In a few cases, we give proofs for facts that have reasonably direct analytic proofs; these results are probably familiar to geometers. We shall need a few results from algebraic geometry besides those cited in this section; they will be given as needed.

We use $\mathbf{F}$ to refer to either $\mathbf{R}$ or $\mathbf{C}$. We generally regard $\mathbf{R}^{n}$ as a subset of $\mathbf{C}^{n}$ (in the obvious way); thus we speak of the (complex) Zariski closure of a variety 
in $\mathbf{R}^{n}$. We generally use a subscript "0" to refer to subsets of $\mathbf{R}^{n}$. If $X$ is any subset of $\mathbf{C}^{n}$, its (complex) Zariski closure is denoted by $X^{\mathbf{C}}$; if $X_{0} \subset \mathbf{R}^{n}$, its Zariski closure in $\mathbf{R}^{n}$ is denoted by $X_{0}^{\mathbf{R}}$. When we are dealing with $\mathbf{F}$, we denote the Zariski closure of a set $S$ by $S^{\#}$.

(3.1) Lemma. For any $X_{0} \subset \mathbf{R}^{n}$,

(i) $\left(X_{0}^{\mathbf{R}}\right)^{\mathbf{C}}=X_{0}^{\mathbf{C}}$;

(ii) $X_{0}^{\mathbf{C}} \cap \mathbf{R}^{n}=X_{0}^{\mathbf{R}}$;

(iii) $X_{0}^{\mathrm{R}}$ irreducible in $\mathbf{R}^{n} \Rightarrow X_{0}^{\mathrm{C}}$ irreducible in $\mathbf{C}^{n}$.

(Recall that $X$ is irreducible if it is not the union of two proper subvarieties.) These are all easy, so we omit the proofs.

Let $X_{0} \subseteq \mathbf{R}^{n}$ be a variety (hence Zariski-closed), and let

$$
I_{0}=\left\{p \in \mathbf{R}\left[X_{1}, \ldots, X_{n}\right]: p \mid X_{0} \equiv 0\right\} .
$$

Then $X$, the Zariski-closure of $X_{0}$ in $\mathbf{C}^{n}$, is the set of common zeros of $I_{0} \subset$ $\mathbf{C}\left[X_{1}, \ldots, X_{n}\right]$; indeed, the polynomials vanishing on $X$ are just the elements of $I=I_{0} \otimes_{\mathbf{R}} \mathbf{C}$. (Simply notice that any $p \in \mathbf{C}\left[X_{1}, \ldots, X_{n}\right]$ can be written uniquely as $p_{1}+i p_{2}$, with $p_{j} \in \mathbf{R}\left[X_{1}, \ldots, X_{n}\right]$, and that $\left.\left.p\right|_{X_{0}} \equiv 0 \Leftrightarrow p_{1}\right|_{X_{0}},\left.p_{2}\right|_{X_{0}}$ are both 0.)

Let $X \subseteq \mathbf{C}^{n}$ be an irreducible algebraic variety. Then $X$ is a finite disjoint union of complex manifolds, $X=S_{1} \cup \cdots \cup S_{k}$, where each $S_{j}$ is Zariski-open in the complement of $X \backslash\left(S_{1} \cup \cdots \cup S_{j-1}\right)$ for all $j$. We define $\operatorname{dim}_{\mathrm{C}} X$ to be the maximum of the $\operatorname{dim}_{\mathrm{C}} S_{i}$. This coincides with the usual algebraic definition of the dimension of a variety, and is independent of the decomposition of $X$ given above. One useful fact is

(3.2) Any subvariety of $X$ has lower dimension than $X$. (See, e.g., $[4$, p. 6]). Thus, in particular, we have

$$
\operatorname{dim} X=\operatorname{dim} S_{1}>\operatorname{dim}\left(S_{2} \cup \cdots \cup S_{k}\right) .
$$

For algebraic varieties in $\mathbf{R}^{n}$, there are similar results. We shall need a more general fact. A semialgebraic set in $\mathbf{R}^{n}$ is a set $S$ in the Boolean algebra generated by the sets $\{x: p(x) \geq 0\},\{x: p(x)=0\}$, where $p$ is a polynomial. (Observe that for any given semialgebraic set, only finitely many polynomials play a role.) Given semialgebraic sets $T_{1}, \ldots, T_{r}$, a stratification of $\left(T_{1}, \ldots, T_{r}\right)$ is a partition $P=$ $\left\{S_{1}, \ldots, S_{m}\right\}$ of $\mathbf{R}^{n}$ such that

(i) each $S_{i}$ is a connected embedded manifold in $\mathbf{R}^{n}$ (the manifold topology $=$ the relative topology);

(ii) for every $x \in \mathbf{R}^{n}, \exists$ a neighborhood $N_{x}$ of $\mathbf{R}^{n}$ such that $N_{x} \cap S_{i}$ is connected (or empty), $1 \leq i \leq m$;

(iii) $S_{i} \cap S_{j}^{-} \neq \varnothing \Rightarrow S_{i} \subseteq S_{j}^{-}$(the closure is in the Euclidean topology);

(iv) each $S_{j}$ is semialgebraic;

(v) each $T_{i}$ is the union of the $S_{j}$ meeting it.

Such stratifications always exist; see [8]. It is not hard to see that if $P$ is a stratification of the semialgebraic set $T$, then $\max \left(\operatorname{dim} S_{j}: S_{j} \in \mathcal{P}\right)$ is independent of $\mathcal{P}$; we define $\operatorname{dim}_{\mathbf{R}} T$ to be this number. Furthermore, there is a unique natural measure class $[\nu]$ on $T$. This is obtained as follows: let $\operatorname{dim} T=k$. On each $S_{j}$ with $\operatorname{dim} S_{j}=k$, use a nonvanishing $k$-form to get a measure $\nu_{j}$; if $\operatorname{dim} S_{j}<k$, define 
$\nu_{j}$ on $S_{j}$ to be 0 . Now let $\nu=\sum \nu_{j}$. It is easy to see that $[\nu]$ is independent of the choice of $k$-forms and of the stratification.

The following proposition lets us compare dimensions; it is close to one in Appendix IV of [10].

(3.3) PROPOSITION. Let $X_{0}$ be an irreducible algebraic variety in $\mathbf{R}^{n}$; let $X$ be its Zariski-closure in $\mathbf{C}^{n}$. Then

(i) $X$ is irreducible;

(ii) any subvariety of $X$ or $X_{0}$ has lower dimension;

(iii) $\operatorname{dim}_{\mathbf{R}} X_{0}=\operatorname{dim}_{\mathbf{C}} X$.

Proof. We noted (i) in Lemma 3.1. Statement (ii) for $X$ is just (3.2); by (3.1)(ii) it follows for varieties over $\mathbf{R}$ once we prove (iii). Thus we need only prove (iii).

In view of (ii), the decomposition of $X$ into complex manifolds has a single piece $U$ of largest dimension; set $m=\operatorname{dim}_{\mathbf{C}} U$. If $U \cap X_{0}=\varnothing$, then $X \backslash U$ is Zariski-closed and contains $X_{0}$; this contradicts the definition of $X$ as the Zariski-closure of $X_{0}$. Let $x \in U \cap X_{0}$, and let $I$ be the prime ideal of polynomials vanishing on $X$. From Corollary 1.20 of [4], there are polynomials $f_{1}, \ldots, f_{k} \in I(k=n-m)$ such that the $f_{j}$ have linearly independent linear terms and

$$
I=\left\{f \in \mathbf{C}[X]: f=\sum_{j=1}^{k} \frac{h_{j}}{g} f_{j}, h_{j}, g \in \mathbf{C}\left[X_{1}, \ldots, X_{n}\right], g(x) \neq 0\right\} .
$$

We may assume that the linear terms of the $f_{j}$ are real; from the proof in [4] we may further assume (taking real parts) that the $f_{j}$ are in $\mathbf{R}\left[X_{1}, \ldots, X_{n}\right]$. The Implicit Function Theorem now gives a real manifold of dimension $m$ in $X \cap \mathbf{R}^{n}=X_{0}$ and containing $x$; hence $\operatorname{dim}_{\mathbf{R}} X_{0} \geq \operatorname{dim}_{\mathbf{C}} X$. The other inequality is also an easy consequence of the Implicit Function Theorem.

Let $S \subseteq \mathbf{F}^{m}$; let $\varphi: S \rightarrow \mathbf{F}^{n}$ be a map. We say that $\varphi$ is rational nonsingular if there are Zariski-open sets $U_{\theta} \subseteq \mathbf{F}^{m}$ and explicit rational maps $\varphi_{\theta}$ on $U_{\theta}$,

$$
\begin{aligned}
\varphi_{\theta}\left(z_{1}, \ldots, z_{m}\right)=\varphi_{\theta}(z)=\left(\frac{r_{1}(z)}{s_{1}(z)}, \ldots, \frac{r_{n}(z)}{s_{n}(z)}\right), \\
r_{i}, s_{i} \in \mathbf{F}\left[z_{1}, \ldots, z_{m}\right], s_{i} \neq 0 \text { on } U_{\theta},
\end{aligned}
$$

such that (i) $\varphi_{\theta}=\varphi$ on $S \cap U_{\theta}$ and (ii) the $U_{\theta}$ cover $S$.

(3.4) LEMMA. Let $S \subseteq \mathbf{F}^{m}$; let $\varphi: S \rightarrow \mathbf{F}^{n}$ be rational nonsingular on $S$, and let $T=\varphi(S)$. Then $\varphi: S \rightarrow T$ is continuous when both sets have the relative Zariski topologies.

PrOOF. We begin with two reductions.

(i) It suffices to show that for any rational nonsingular map $\varphi: S \rightarrow \mathbf{F}^{n}, \varphi^{-1}(0)$ is Zariski-closed in $S$. For if $A$ is any Zariski-closed set in $T$, then there are finitely many polynomials $P_{j}, 1 \leq j \leq d$, such that $A$ is the set of common zeros of the $p_{j}$. Let $p=\left(p_{1}, \ldots, p_{d}\right): \mathbf{F}^{n} \rightarrow \mathbf{F}^{d}$; then $p \circ \varphi$ is rational nonsingular on $S$, and $\varphi^{-1}(A)=(p \circ \varphi)^{-1}(0)$. 
Thus we let $X=\varphi^{-1}(0) \subseteq S$; let $X^{\#}$ be the Zariski closure of $X$ in $\mathbf{F}^{m}$. It suffices to prove

(ii) If $X^{\#}$ is irreducible, then $X$ is Zariski-closed in $S$.

For we can write $X^{\#}=A_{1} \cup \cdots \cup A_{r}$ with the $A_{j}$ Zariski-closed and irreducible, and $A_{i} \not \subset A_{j}$ if $i \neq j$. Set $X_{j}=X \cap A_{j}$; we may assume that each $X_{j}$ is nonempty. Then $\bigcup_{j} X_{j}^{\#}=X^{\#}$, since it is closed, covers $X$, and is contained in $X^{\#}$. For fixed $i$, we thus have $\bigcup_{j} X_{j}^{\#} \cap A_{i}=A_{i}$. As $A_{i}$ is irreducible, we must have $X_{j}^{\#} \cap A_{i}=A_{i}$ for some $j ; j=i$, since $X_{j}^{\#} \cap A_{i} \subseteq A_{j} \cap A_{i} \neq A_{i}$ if $j \neq i$. So $X=\bigcup_{j} X_{j}$, where each $X_{j}^{\#}$ is irreducible. Let $g_{1}, \ldots, g_{s}$ be polynomials on $\mathbf{F}^{m}$ such that $X_{1}^{\#}$ is the set of common zeros of the $g_{j}$; let $\tilde{\varphi}=\left(\varphi ; g_{1}, \ldots, g_{s}\right): \mathbf{F}^{m} \rightarrow \mathbf{F}^{n+s}$. Now $S \cap \tilde{\varphi}^{-1}(0)=X_{1} ; X_{1}^{\#}$ is irreducible, and (ii) implies that $X_{1}$ is Zariski-closed in $S$. Similarly, the other $X_{j}$ are Zariski-closed in $S$.

We now prove (ii); thus we assume that $X^{\#}$ is irreducible. Let $\varphi_{\theta}, U_{\theta}$ cover $S$ as above, and let $I=\left\{\theta: U_{\theta} \cap X \neq \varnothing\right\}$. Fix $\theta \in I$ and write $\varphi_{\theta}=\left(r_{1} / s_{1}, \ldots, r_{n} / s_{n}\right)$ with $s_{i} \neq 0$ on $U_{\theta}$ and $\varphi_{\theta}=\varphi$ on $S \cap U_{\theta}$. Let $A_{\theta}$ be the set of common zeros of the $r_{i}$. Then $X \cap U_{\theta}=S \cap U_{\theta} \cap A_{\theta}$. Clearly $X \subseteq \bigcup_{\theta \in I} A_{\theta}$ and the $A_{\theta}$ are Zariski-closed in $\mathbf{F}^{m}$. By the irreducibility of $X^{\#}$, there is some $\theta_{0}$ with $X \subseteq A_{\theta_{0}}$. Suppose that $\theta \in I$; then $X \cap U_{\theta} \cap U_{\theta_{0}}$ is nonempty Zariski-dense in $X^{\#}$. Let

$$
\varphi_{\theta_{0}}=\left(r_{1} / s_{1}, \ldots, r_{n} / s_{n}\right), \quad \varphi_{\theta}=\left(r_{1}^{\prime} / s_{1}^{\prime}, \ldots, r_{n}^{\prime} / s_{n}^{\prime}\right) \text {. }
$$

Then on $X \cap U_{\theta} \cap U_{\theta_{0}}$, we have

$$
\begin{array}{ll}
\text { (a) } r_{i} s_{i}^{\prime}=r_{i}^{\prime} s_{i}=0, & \text { all } i \\
\text { (b) } s_{i} \neq 0, \quad s_{i}^{\prime} \neq 0, & \text { all } i .
\end{array}
$$

Then (3.5a) holds in $X^{\#}$, by density. Because $X \subseteq A_{\theta_{0}}$, the $r_{i}$ are identically 0 on $X$; hence the $r_{i}^{\prime}$ are 0 on $X \cap U_{\theta} \cap U_{\theta_{0}}$. Therefore

$$
\varnothing \neq\left(X \cap U_{\theta} \cap U_{\theta_{0}}\right) \subseteq A_{\theta}, \quad \forall \theta \in I ;
$$

the irreducibility of $X^{\#}$ gives

$$
\left(X \cap U_{\theta} \cap U_{\theta_{0}}\right)^{\#}=X^{\#} \subseteq A_{\theta}, \quad \forall \theta \in I .
$$

We also have

$$
S \cap A_{\theta} \cap U_{\theta} \subseteq X, \quad \forall \theta \in I
$$

as noted above.

Now let $x \in X^{\#} \cap S$. Since $X \cap U_{\theta} \neq \varnothing \Leftrightarrow X^{\#} \cap U_{\theta} \neq \varnothing$, we have $X^{\#} \subseteq \bigcup_{\theta \in I} U_{\theta}$. Thus there exists $\theta \in I$ with $x \in U_{\theta}$. From (3.6), $x \in A_{\theta}$. Thus $x \in A_{\theta} \cap U_{\theta} \cap S \subseteq X$, so that $X^{\#} \cap S \subseteq X$. This proves that $X$ is Zariski-closed in $S$.

We note two corollaries.

(3.7) COROLLARY. If $\varphi: S \rightarrow T$ is birational nonsingular, then it is a Zariski homeomorphism.

(3.8) COROLLARY. If $\varphi: S \rightarrow \mathbf{F}^{n}$ is rational nonsingular, and if $S^{\#} \subseteq \mathbf{F}^{m}$ is irreducible, then $\varphi(S)^{\#}$ is irreducible.

ProOF. Let $\varphi(s)=T$. If $T^{\#}$ is not irreducible, then $T=X_{1} \cup X_{2}$ with $X_{1}, X_{2}$ Zariski-closed in $T$. In that case, $\varphi^{-1}\left(X_{1}\right), \varphi^{-1}\left(X_{2}\right)$ show that $S$ is not irreducible.

We shall also find the following lemmas useful. 
(3.9) LEMMA. Suppose that $\varphi: S \rightarrow T$ is a birational nonsingular map $(S \subseteq$ $\left.\mathbf{F}^{m}, T \subseteq \mathbf{F}^{n}\right)$. Assume that

(a) $T$ is Zariski-open in its closure: $T=B^{c} \cap T^{\#}$ for some Zariski-closed $B \subseteq$ $\mathbf{F}^{n}\left(B^{c}=\right.$ complement of $\left.B\right)$;

(b) there is an irreducible closed variety $E \subseteq \mathrm{F}^{m}$ such that $S \cap E$ contains a nonempty Zariski-open subset of $E$.

Let $W=\varphi(S \cap E)$. Then $W^{\#}$ is irreducible in $\mathbf{F}^{n}$ and $W$ contains a subset $A$ that is Zariski-open and dense in $W^{\#}$.

PROOF. The restriction of $\varphi$ to $S \cap E$ is rational nonsingular; $(S \cap E)^{\#}=E$ is irreducible by assumption. Corollary 3.8 now says that $W^{\#}$ is irreducible. Let $\psi=\varphi^{-1}: T \rightarrow S$; let $p=\left(p_{1}, \ldots, p_{d}\right): \mathbf{F}^{m} \rightarrow \mathbf{F}^{d}$ be polynomial, with $p^{-1}(0)=E$. Because $\psi$ is rational nonsingular, there are Zariski-open subsets $U_{\theta} \subseteq \mathbf{F}^{n}$ covering $T$, together with rational maps $\psi_{\theta}$, nonsingular on $U_{\theta}$, such that $\psi_{\theta}=\psi$ on $T \cap U_{\theta}$. Then $p \circ \psi: T \rightarrow \mathbf{F}^{d}$ is rational nonsingular and is given on $T \cap U_{\theta}$ by $g_{\theta}=p \circ \psi_{\theta}$ :

$$
g_{\theta}=\left(\frac{r_{1}}{s_{1}}, \ldots, \frac{r_{d}}{s_{d}}\right) \quad \text { on } U_{\theta} \text { with } s_{i} \neq 0 \text { on } U_{\theta} ; r_{i}, s_{i} \in \mathbf{F}\left[t_{1}, \ldots, t_{n}\right] \text {. }
$$

Let $A_{\theta}=\left\{x \in \mathbf{F}^{n}: r_{i}(x)=0,1 \leq i \leq d\right\}$ and $G_{\theta}=\left\{x \in U_{\theta}: g_{\theta}(x)=0\right\}$. Then $A_{\theta}$ is Zariski-closed in $\mathbf{F}^{n}$ and $G_{\theta}=A_{\theta} \cap U_{\theta}$. Now

$$
\begin{gathered}
W=(p \circ \psi)^{-1}(0)=T \cap(p \circ \psi)^{-1}(0) \quad(T=\operatorname{dom} \psi) ; \\
W \cap U_{\theta}=U_{\theta} \cap(p \circ \psi)^{-1}(0)=G_{\theta} \cap U_{\theta} \cap T=A_{\theta} \cap U_{\theta} \cap T .
\end{gathered}
$$

We know from (i) that $T=B^{c} \cap T^{\#}$ for some Zariski-closed $B \subseteq \mathbf{F}^{n}$. Hence

$$
W \cap U_{\theta}=B^{c} \cap T^{\#} \cap G_{\theta} \cap U_{\theta}=B^{c} \cap T^{\#} \cap A_{\theta} \cap U_{\theta} .
$$

Let $I=\left\{\theta: U_{\theta}\right.$ meets $\left.W\right\}=\left\{\theta: U_{\theta}\right.$ meets $\left.W^{\#}\right\}$. Then for $\theta \in I$,

$$
\begin{gathered}
W \cap U_{\theta}=T \cap(p \circ \psi)^{-1}(0) \cap U_{\theta} ; \\
W^{\#}=\left(W \cap U_{\theta}\right)^{\#} \subseteq T^{\#} \cap\left((p \circ \varphi)^{-1}(0) \cap U_{\theta}\right)^{\#}=T^{\#} \cap G_{\theta}^{\#} \subseteq T^{\#} \cap A_{\theta} .
\end{gathered}
$$

Hence

$$
W^{\#} \cap U_{\theta} \subseteq T^{\#} \cap A_{\theta} \cap U_{\theta}, \quad \forall \theta \in I .
$$

Comparing (3.10) and (3.11) gives

$$
W^{\#} \cap U_{\theta} \cap B^{c} \subseteq B^{c} \cap T^{\#} \cap A_{\theta} \cap U_{\theta}=W \cap U_{\theta} \subseteq W^{\#} \cap U_{\theta}, \quad \forall \theta \in I .
$$

Since the $U_{\theta}$ cover $W^{\#}$, we get (summing over $\theta \in I$ ) $W^{\#} \cap B^{c}=W \subseteq W^{\#}$. So we can take $A=W^{\#} \cap B^{c}=W$.

We conclude this section by recalling an alternative version of the Multiplicity Theorem 2.2, valid in the case where $\operatorname{dim}_{\chi}=1$. It is simpler to prove than Theorem 2.2 because it stops short of describing multiplicities canonically. It is essentially established as Theorem 4 of [2], well before one meets the most difficult technical details involved in Theorem 2.2.

(3.12) Theorem (Noncanonical Multiplicity Formula). Let $k_{0}$ be a Lie subalgebra of a real Lie algebra $\mathfrak{g}_{0}$, and let $K_{0} \subseteq G_{0}$ be the corresponding Lie group. Suppose that $\chi=e^{2 \pi i f} \in K_{0}^{\hat{0}}$ is a 1-dimensional unitary representation; 
let $\rho=\operatorname{Ind}(K \uparrow G, \chi)$. Fix a Malcev basis in $\mathfrak{g}_{0}$ and form the corresponding $\mathcal{E}$ layering of $\mathfrak{g}_{0}^{*}$. Let $e \in \mathcal{E}$ be the first index such that $U_{e}$ meets $f^{\prime}+\mathfrak{k}_{0}^{\perp}$; and let $P_{e}: U_{e} \rightarrow \Sigma_{e} \times V_{S(e)}$ be the (rational) inverse of the parametrizing map. Then

(i) $e$ is also the first index such that $U_{e}$ meets $f^{\prime}+E_{1}^{0}$, and their intersection is Zariski-open in $f^{\prime}+E_{1}^{0}$. (The subspace $E_{1}^{0}$ is the one of Proposition 2.5.)

Let $S_{1}^{0}=U_{e} \cap\left(f^{\prime}+E_{1}^{0}\right)$; define $\varphi: S_{1}^{0} \rightarrow \Sigma_{e} \subseteq V_{T(e)}$ by $\varphi=\left.\pi_{T} \circ P_{e}\right|_{S_{1}^{0}}$, where $\pi_{T}$ is the projection onto $V_{T(e)}$ killing $V_{S(e)}$. Then

(ii) $\Sigma_{1}^{0}=\varphi\left(S_{1}^{0}\right)$ is semialgebraic. If $\left[\nu_{1}\right]$ is its canonical measure class, then

$$
\rho \cong \int_{\Sigma_{1}^{0}}^{\oplus} n(l) \pi_{l} d \nu_{1}(l),
$$

where either $n(l)=+\infty\left(\right.$ when $\left.\tau_{0}>0\right)$ or

$$
n(l)=\operatorname{Card}\left(G_{0} \cdot l \cap\left(f^{\prime}+E_{1}^{0}\right)\right), \quad \text { all } l \in \Sigma_{1}^{0}\left(\text { when } \tau_{0}=0\right) .
$$

In the latter case, $n(l)$ is uniformly bounded, $\nu_{1}$-almost everywhere on $\Sigma_{1}^{0}$.

REMARKS. 1. The result is a geometric multiplicity formula, but the variety $f^{\prime}+E_{1}^{0}$ is not canonically defined.

2. If $\tau_{0}>0$, then $\operatorname{Card}\left(G_{0} \cdot l \cap\left(f^{\prime}+E_{1}^{0}\right)\right)=\infty$; thus (3.14) holds regardless of the size of $\tau_{0}$. (See Theorem 2.2 for the definition of $\tau_{0}$.)

4. This section is devoted to various results on coadjoint orbits for complex nilpotent Lie groups and algebras. Many of the proofs are essentially the same as for real groups (for these we refer to [2]), and many others are elementary consequences of the definitions. We often use a subscript "zero" to indicate a real Lie algebra.

Let $\mathfrak{g}$ be a complex nilpotent Lie algebra. There is a corresponding simply connected complex Lie group $G$, which acts on $\mathfrak{g}$ via the adjoint action. If $\mathfrak{g}_{\mathbf{R}}=\mathfrak{g}$ with the field reduced to $\mathbf{R}, \operatorname{Ad} G$ acts on $\mathfrak{g}_{\mathbf{R}}$ as well. There are two corresponding contragredient actions $\operatorname{Ad}^{*}(G)$, one on $\mathfrak{g}^{*}=\operatorname{Hom}_{\mathbf{C}}(\mathfrak{g}, \mathbf{C})$ via $\mathbf{C}$-linear maps, and one on $\mathfrak{g}_{\mathbf{R}}^{*}=\operatorname{Hom}_{\mathbf{R}}\left(\mathfrak{g}_{\mathbf{R}}, \mathbf{R}\right)$ via $\mathbf{R}$-linear maps.

(4.1) LEMMA. Define $J: \mathfrak{g}_{\mathbf{R}}^{*} \rightarrow \mathfrak{g}^{*}$ by

$$
(J f)(Z)=f(Z)-i f(i Z), \quad Z \in \mathfrak{g} .
$$

Then $J$ is an $\mathbf{R}$-linear isomorphism that intertwines the actions of $\operatorname{Ad}^{*}(G)$. Its inverse is given by

$$
\left(J^{-1} \varphi\right)(Z)=\operatorname{Re}(\varphi(Z)), \quad \forall \varphi \in \mathfrak{g}^{*}, \forall Z \in \mathfrak{g}_{\mathbf{R}} .
$$

PROOF. If $\varphi \in \mathfrak{g}^{*}$, write $\varphi=\varphi_{1}+i \varphi_{2}$, where $\varphi_{1}(Z)=\operatorname{Re} \varphi(Z)$ and $\varphi_{2}(Z)=$ $\operatorname{Im} \varphi(Z)$. Then $\varphi_{1}, \varphi_{2} \in \mathfrak{g}_{\mathbf{R}}^{*}$. Moreover,

$$
\varphi_{1}(i Z)+i \varphi_{2}(i Z)=\varphi(i Z)=i \varphi(Z)=-\varphi_{2}(Z)+i \varphi_{1}(Z)
$$

so that

$$
\varphi_{2}(Z)=-\varphi_{1}(i Z), \quad \forall Z \in \mathfrak{g} .
$$

This shows that $\varphi=J \varphi_{1}$, which proves the second claim. If $x \in G$ and $z \in \mathfrak{g}$, then

$$
\begin{aligned}
\left(\left(\operatorname{Ad}^{*} x\right)^{-1} \varphi\right)(Z) & =\varphi((\operatorname{Ad} x) Z)=\varphi_{1}((\operatorname{Ad} x) Z)-i \varphi_{1}(i(\operatorname{Ad} x) Z) \\
& =\varphi_{1}((\operatorname{Ad} x) Z)-i \varphi_{1}((\operatorname{Ad} x)(i Z)) \\
& =\left(\operatorname{Ad}^{*} x^{-1}\left(\varphi_{1}\right)\right)(Z)-i\left(\operatorname{Ad}^{*} x^{-1}\left(\varphi_{1}\right)\right)(i Z) \\
& =\left(J\left(\operatorname{Ad}^{*} x^{-1}\right) \varphi_{1}\right)(Z) .
\end{aligned}
$$


As $\varphi=J \varphi_{1}$, the intertwining property is proved.

The standard proof (in, e.g., [5]) shows that for $\varphi \in \mathfrak{g}^{*}, \operatorname{Stab}_{G}(\varphi)=\{x \in$ $\left.G: \operatorname{Ad}^{*}(x) \varphi=\varphi\right\}$ is a connected Lie subgroup of $G$ whose Lie algebra is $\mathfrak{p}(\varphi)=$ $\{Z \in \mathfrak{g}: \varphi([Z, \mathfrak{g}])=(0)\}$. If $f \in \mathfrak{g}_{\mathbf{R}}^{*}$, then its stabilizer has Lie algebra $\mathfrak{p}(f)=\{Z \in$ $\left.\mathfrak{g}_{\mathbf{R}}: f\left(\left[Z, \mathfrak{g}_{\mathbf{R}}\right]\right)=(0)\right\}$. A simple calculation gives

$$
\mathfrak{p}(J f)=\mathfrak{p}(f), \quad \forall f \in \mathfrak{g}_{\mathbf{R}} .
$$

Hence $\mathfrak{p}(f)$ is always a complex subalgebra of $\mathfrak{g}\left(=\mathfrak{g}_{\mathbf{R}}\right)$. Construct a maximal isotropic complex subalgebra $\mathfrak{m}$ for $J f$. Simple calculations with dimensions prove $\mathfrak{m}_{\mathbf{R}}$ maximal isotropic for $f$; that is,

(4.4) For all $f \in \mathbf{g}_{\mathbf{R}}^{*}, f$ has a maximal isotropic subalgebra that is complex.

This means that every element of $G^{\wedge}$ is induced from a 1-dimensional representation of a complex subgroup. Note also that $\operatorname{codim}_{\mathbf{C}} \mathfrak{p}(f)$ is even, so that $\operatorname{dim}_{\mathbf{R}}\left(\mathfrak{g}_{\mathbf{R}} / \mathfrak{p}(f)\right)$ is divisible by 4 .

We next consider a different situation: let $g_{0}$ be a real nilpotent Lie algebra, with corresponding Lie group $G_{0}$, and let $\mathfrak{g}$ be its complexification. Imbed $\mathfrak{g}_{0}^{*}=$ $\operatorname{Hom}_{\mathbf{R}}\left(\mathfrak{g}_{0}, \mathbf{R}\right)$ into $\mathfrak{g}^{*}$ in the natural way via $I: \mathfrak{g}_{0}^{*} \rightarrow \mathfrak{g}^{*}$ :

$$
I(l)(Z)=I(l)(X+i Y)=l(X)+i l(Y), \quad l \in \mathfrak{g}_{0}^{*}, \quad Z=X+i Y \in \mathfrak{g} .
$$

Then Range $I=\left\{\varphi \in \mathfrak{g}^{*}: \varphi\left(\mathfrak{g}_{0}\right) \subseteq \mathbf{R}\right\}$. If we imbed $\mathfrak{g}_{0}^{*}$ into $\mathfrak{g}_{\mathbf{R}}^{*}$ via

$$
\left(I_{\mathbf{R}} l\right)(Z)=\left(I_{\mathbf{R}} l\right)(X+i Y)=l(X)
$$

then $I=J \circ I_{\mathbf{R}}$ and Range $I_{\mathbf{R}}=\left(i \mathfrak{g}_{0}\right)^{\perp} \subseteq \mathfrak{g}_{\mathbf{R}}^{*}$. Regard $G_{0}$ as a subgroup of $G=\exp (\mathfrak{g})$; then $I_{\mathbf{R}}, J$, and $I$ all commute with the coadjoint actions of $G_{0}$. Furthermore, if $l \in \mathfrak{g}_{0}^{*}$, then $\mathfrak{p}_{l}=\left\{X \in \mathfrak{g}_{0}: l\left(\left[X, \mathfrak{g}_{0}\right]\right)=(0)\right\}$ satisfies

$$
\mathfrak{p}(I l)=\mathfrak{p}_{l} \otimes_{\mathbf{R}} \mathbf{C}=\mathfrak{p}_{l}+i_{\mathfrak{p}_{l}}=\mathfrak{p}\left(I_{\mathbf{R}} l\right) .
$$

If $\mathfrak{m}_{0}$ is a polarizing subalgebra for $l$, then $\mathfrak{m}=\mathfrak{m}_{0}+i \mathfrak{m}_{0}$ is polarizing for both $I l$ and $I_{\mathbf{R}} l$.

We now compare the $\mathcal{E}$-layerings for $\mathfrak{g}^{*}$ and $\mathfrak{g}_{\mathbf{R}}^{*}$ under the action of $G$. Let $\left\{X_{1}, \ldots, X_{n}\right\}$ be a (complex) Malcev basis for $\mathfrak{g}$, with dual basis $X_{1}^{*}, \ldots, X_{n}^{*}$ in $\mathfrak{g}^{*}$. Then $\left\{Y_{1}, \ldots, Y_{2 n}\right\}$, where $Y_{2 j-1}=X_{j}$ and $Y_{2 j}=i X_{j}$, is a (real) Malcev basis for $\mathfrak{g}_{\mathbf{R}}$, and the dual basis $Y_{1}^{*}, \ldots, Y_{2 n}^{*}$ is a Jordan-Hölder basis for $\mathfrak{g}_{\mathbf{R}}^{*}$. Moreover, calculation gives

$$
J\left(Y_{2 j-1}^{*}\right)=X_{j}^{*}, \quad J\left(Y_{2 j}^{*}\right)=-i X_{j}^{*}, \quad 1 \leq j \leq n .
$$

Let

$$
\begin{array}{rlrl}
\mathfrak{g}_{j}^{*} & =\mathbf{C}-\operatorname{span}\left\{X_{j+1}^{*}, \ldots, X_{n}^{*}\right\}, & p_{j}: \mathfrak{g}^{*} \rightarrow \mathfrak{g}^{*} / \mathfrak{g}_{j}^{*}, & 1 \leq j \leq n ; \\
\left(\mathfrak{g}_{\mathbf{R}}^{*}\right)_{j} & =\mathbf{R}-\operatorname{span}\left\{Y_{j+1}^{*}, \ldots, Y_{n}^{*}\right\}, & p_{j}^{\mathbf{R}}: \mathfrak{g}_{\mathbf{R}}^{*} \rightarrow \mathfrak{g}_{\mathbf{R}}^{*} /\left(\mathfrak{g}_{\mathbf{R}}^{*}\right)_{j}, \quad 1 \leq j \leq 2 n .
\end{array}
$$

Then (4.6) says that $J\left(\left(\mathfrak{g}_{\mathrm{R}}^{*}\right)_{2 j}\right)=\mathfrak{g}_{j}^{*}$. We thus get induced $J$-maps $J_{2 j}: \mathfrak{g}_{\mathrm{R}}^{*} /\left(\mathfrak{g}_{\mathrm{R}}^{*}\right)_{2 j}$ $\rightarrow \mathfrak{g}^{*} / \mathfrak{g}_{j}^{*}$; these maps are equivariant for the induced action of $\operatorname{Ad}^{*}(G)$, and we have $p_{j} \circ J=J_{2 j} \circ p_{2 j}^{\mathbf{R}}$.

For $l \in \mathfrak{g}^{*}$, define $e_{j}(l)=\operatorname{dim}_{\mathbf{C}} G \cdot p_{j}(l)$ for $1 \leq j \leq n$, and let $e(l)=$ $\left(e_{1}(l), \ldots, e_{n}(l)\right)$; let $\mathcal{E}=\left\{e: \exists l \in \mathfrak{g}^{*}\right.$ with $\left.e(l)=e\right\} \subset \mathbf{Z}^{n}$. Similarly, for $f \in \mathfrak{g}_{\mathbf{R}}^{*}$, define $e_{j}(f)=\operatorname{dim}_{\mathbf{R}} G \cdot p_{j}^{\mathbf{R}}(l)$ for $1 \leq j \leq 2 n$; set $e(f)=\left(e_{1}(f), \ldots, e_{2 n}(f)\right)$, and let $\mathcal{E}_{\mathbf{R}}=\left\{e: \exists f \in \mathfrak{g}_{\mathbf{R}}^{*}\right.$ with $\left.e(f)=e\right\} \subset \mathbf{Z}^{2 n}$. 
(4.7) LEMMA. Let notation be as above. For $e \in \mathcal{E}_{\mathbf{R}}$, define $\alpha(e) \in \mathbf{R}^{n}$ by $\alpha(e)_{j}=\frac{1}{2} e_{2 j}$. Then $\alpha(e) \in \mathcal{E}$, and the map $e \mapsto \alpha(e)$ is a bijection of $\mathcal{E}_{\mathbf{R}}$ onto $\mathcal{E}$. If $e^{\prime}=\left(e_{1}, \ldots, e_{n}\right) \in \mathcal{E}$, then $\alpha^{-1}\left(e^{\prime}\right)=\left(e_{1}, \ldots, e_{2 n}\right)$, with $e_{2 j}=2 e_{j}^{\prime}, e_{2 j-1}=$ $e_{j}^{\prime}+e_{j-1}^{\prime}\left(\right.$ here $\left.e_{0}^{\prime}=0\right)$. Moreover,

$$
e(J f)=\alpha(e(f)), \quad \forall f \in \mathfrak{g}_{\mathbf{R}}^{*}
$$

ProOF. We have

$$
G \cdot p_{j}(J f)=J\left(G \cdot p_{2 j}^{\mathbf{R}}(f)\right), \quad \forall f \in \mathfrak{g}_{\mathbf{R}}^{*} \text { and } \forall j, 1 \leq j \leq n,
$$

as noted earlier. Hence

$e_{j}(J f)=\operatorname{dim}_{\mathbf{C}}\left(G \cdot p_{j}(J f)\right)=\frac{1}{2} \operatorname{dim}_{\mathbf{R}}\left(G \cdot p_{j}(J f)\right)=\frac{1}{2} \operatorname{dim}_{\mathbf{R}}\left(G \cdot p_{2 j}^{\mathbf{R}}(f)\right)=\frac{1}{2} e_{2 j}(f)$,

from which (4.8) follows. This implies that $\alpha$ is a bijection. To verify the formula $\alpha^{-1}\left(e^{\prime}\right)$, note that $e_{2 j}=2 e_{j}^{\prime}$ by the formula for $\alpha$; as for the odd terms, their value is determined by the fact that $e_{i+1}-e_{i}=0$ or 1 for all $i$ if $e \in \mathcal{E}_{\mathbf{R}}$ (see Theorem 2.1).

It follows from (4.8) that if we define layers $U_{e}^{\mathbf{R}}, U_{e}$ in the usual way for $e$ in $\varepsilon^{\mathbf{R}}, \mathcal{E}$, then

$$
J\left(U_{e}^{\mathbf{R}}\right)=U_{\alpha(e)}, \quad \forall e \in \mathcal{E}^{\mathbf{R}} .
$$

Furthermore, Theorem 2.1(ii) refers to an ordering of indices in $\mathcal{E}^{\mathbf{R}}$. This ordering is explicitly described in [2], or in §III.1.1 of [1]. There is a similar ordering for $\mathcal{E}$, and it is not hard to see that one can choose the orderings so that $\alpha$ is order-preserving.

Now fix $e \in \mathcal{E}^{\mathbf{R}}$ and let $\varepsilon=\alpha(e)$. Consider the rational nonsingular parametrizing maps of Theorem 2.1:

$$
\Sigma_{\varepsilon} \times V_{S(\varepsilon)} \underset{Q_{e}}{\stackrel{P_{e}}{\leftrightarrows}} U_{\varepsilon}, \quad \Sigma_{e} \times V_{S(e)} \underset{Q_{\mathbf{R}, e}}{\stackrel{P_{\mathbf{R}, e}}{\leftrightarrows}} U_{e}^{\mathbf{R}} .
$$

From the formula for $\alpha^{-1}$, we see that

$$
S(\varepsilon)=\left\{j_{1}, \ldots, j_{k}\right\} \Leftrightarrow S(e)=\left\{2 j_{1}-1,2 j_{1}, \ldots, 2 j_{k}-1,2 j_{k}\right\}
$$

and similarly for $T(e), T(\varepsilon)$. By (4.6),

$$
V_{S(\varepsilon)}=J\left(V_{S(e)}\right), \quad V_{T(\varepsilon)}=J\left(V_{T(e)}\right) .
$$

Since $J\left(U_{e}^{\mathbf{R}}\right)=U_{\varepsilon}$, we get

$$
\Sigma_{\varepsilon}=U_{\varepsilon} \cap V_{T(\varepsilon)}=J\left(U_{e} \cap V_{T(e)}\right)=J\left(\Sigma_{e}\right) .
$$

In fact, we have $P_{e} \circ J=J \circ P_{\mathbf{R}, e}$; this follows from the geometric description of $P_{e}$ and $P_{\mathbf{R}, e}$,

$$
P_{e}(l)=\left(G \cdot l \cap V_{T(e)}, \pi_{S}(l)\right)
$$

(and similarly for $P_{\mathbf{R}, e}$ ), given in Theorem 2.1 .

5. In this section, we prove Theorem 1.1. We use the notation of $\S \S 3$ and 4 .

From (4.4), we may assume that $\chi$ is 1-dimensional, $\chi=e^{2 \pi i f_{0}}$ on $K$, where $f_{0} \in \mathfrak{k}_{\mathbf{R}}^{*}=\operatorname{Hom}_{\mathbf{R}}\left(\mathfrak{k}_{\mathbf{R}}, \mathbf{R}\right)$ is a Lie algebra homomorphism. It is easy to verify that $\varphi_{0}=J f_{0} \in \operatorname{Hom}_{\mathbf{C}}(\boldsymbol{k}, \mathbf{C})$ is a Lie algebra homomorphism. It is also easy to verify that the $J$-maps have the following properties: 
(i) If $P, P_{\mathbf{R}}$ are the natural projections, then

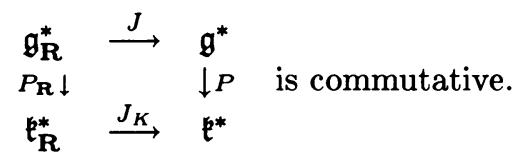

(ii) If $\mathfrak{k}_{\mathbf{R}}^{\perp}=\left\{f \in \mathfrak{g}_{\mathbf{R}}^{*}:\left.f\right|_{\mathfrak{k}_{\mathbf{R}}} \equiv 0\right\}, \mathfrak{k}^{\perp}=\left\{\varphi \in \mathfrak{g}^{*}:\left.\varphi\right|_{\mathfrak{k}} \equiv 0\right\}$, then $J\left(\mathfrak{k}_{\mathbf{R}}^{\perp}\right)=\mathfrak{k}^{\perp}$.

(iii) If $S$ is any subset of $\mathfrak{k}_{\mathbf{R}}^{*}$, then $J P_{\mathbf{R}}^{-1}(S)=P^{-1} J_{K}(S)$. In particular, if $f^{\prime} \in \mathfrak{g}_{\mathbf{R}}^{*}$ satisfies $\left.f^{\prime}\right|_{\mathfrak{k}}=f_{0}$, and if similarly $\left.\varphi^{\prime}\right|_{\mathfrak{k}}=\varphi_{0}$, then $O_{\chi}=\left\{f_{0}\right\}$ and $J$ maps $P_{\mathbf{R}}^{-1}\left(O_{\chi}\right)=f^{\prime}+k_{\mathbf{R}}^{\perp}$ to $P^{-1}\left(\varphi_{0}\right)=\varphi^{\prime}+\mathfrak{k}^{\perp}$. These sets are $K$-invariant, and $J$ maps $K$-orbits to $K$-orbits.

We need to modify the Multiplicity Theorem 3.12 so that it refers to elements of $\mathfrak{g}^{*}$ rather than elements of $\mathfrak{g}_{\mathbf{R}}^{*}$. Let $U_{e}$ be the first layer of $\mathfrak{g}_{\mathbf{R}}^{*}$ that meets $f^{\prime}+\mathfrak{k}_{\mathbf{R}}$, and let $\alpha(e)=\varepsilon$. Then (4.9) and (iii) above imply that $U_{\varepsilon}$ is the first layer of $\mathfrak{g}^{*}$ meeting $\varphi^{\prime}+\mathfrak{k}^{\perp}$. To use Theorem 3.12, however, we also need to describe $J\left(f^{\prime}+E_{1}^{\mathbf{R}}\right)$, where $E_{1}^{\mathbf{R}}$ is the subspace of $\mathfrak{k}_{\mathbf{R}} \mathbf{r}$ appearing in the multiplicity formula. This subspace was produced by examining a different layering, adapted to the pair $\mathfrak{k}_{\mathbf{R}}, \mathfrak{g}_{\mathbf{R}}$. Here are the details we need.

Comparison of $D$-layerings. Starting with $\mathfrak{k} \subseteq \mathfrak{g}$, we can find complex subalgebras $\mathfrak{k}=\mathfrak{g}_{0} \subset \mathfrak{g}_{1} \subset \mathfrak{g}_{2} \subset \cdots \subset \mathfrak{g}_{r}=\mathfrak{g}$ with $\operatorname{dim}_{\mathbf{C}}\left(\mathfrak{g}_{j} / \mathfrak{g}_{j-1}\right)=1$. Choose $X_{j} \in \mathfrak{g}_{j} \backslash \mathfrak{g}_{j-1}$ and define dual vectors $X_{j}^{*} \in \mathfrak{g}^{*}$ such that $X_{j}^{*}\left(X_{k}\right)=\delta_{j k}, X_{j}^{*}(\mathfrak{k})=(0)$. Thus the $X_{j}^{*}$ form a (complex) basis for $\mathfrak{k}^{\perp}$. Let $G_{j}=\exp \left(\mathfrak{g}_{j}\right)$ be the corresponding Lie subgroups to the $\mathfrak{g}_{j}$ in $G$. Then define vectors $Y_{1}, \ldots, Y_{2 r}$ in $\mathfrak{g}_{\mathrm{R}}$ by $Y_{2 j-1}=X_{j}, Y_{2 j}=i X_{j}$. These form an $\mathbf{R}$-basis for $\mathfrak{g}_{\mathbf{R}}$ above $\mathfrak{k}_{\mathbf{R}}$, and, for $1 \leq j \leq 2 r$,

$$
\mathfrak{h}_{j}=\mathfrak{k}_{\mathbf{R}}+\mathbf{R}-\operatorname{span}\left\{Y_{1}, \ldots, Y_{j}\right\}
$$

is a subalgebra of $\mathfrak{g}_{\mathbf{R}}$, with $\operatorname{dim}_{\mathbf{R}}\left(\mathfrak{h}_{j} / \mathfrak{h}_{j-1}\right)=1$. Let $H_{j}=\exp \mathfrak{h}_{j}$. Then $\left(\mathfrak{g}_{j}\right)_{\mathbf{R}}=\mathfrak{h}_{2 j}$ as real Lie algebras, and $\mathfrak{h}_{2 j}^{*}=\left(\mathfrak{g}_{j}\right)_{\mathbf{R}}^{*}$. If $p_{j}: \mathfrak{g}^{*} \rightarrow \mathfrak{h}_{j}^{*}$ and $p_{j}^{\mathbf{R}}: \mathfrak{g}_{\mathbf{R}}^{*} \rightarrow \mathfrak{h}_{j}^{*}$ are the natural projections, and if $J_{j}:\left(\mathfrak{g}_{j}\right)_{\mathbf{R}}^{*} \rightarrow \mathfrak{g}_{j}^{*}$ is defined similarly to $J$, then we have $p_{j} \circ J=J_{j} \circ p_{2 j}^{\mathbf{R}}$. Moreover, the map $J_{j}$ intertwines the actions of $G_{j}=H_{2 j}$ on $\mathfrak{g}_{j}^{*}, \mathfrak{h}_{2 j}^{*}$, and we have

(5.2) $\operatorname{dim}_{\mathbf{C}} G_{j} \cdot p_{j}(J f)=\frac{1}{2} \operatorname{dim}_{\mathbf{R}} G_{j} \cdot p_{2 j}^{\mathbf{R}}(f)=\frac{1}{2} \operatorname{dim}_{\mathbf{R}} H_{2 j} \cdot p_{2 j}^{\mathbf{R}}(f), \quad \forall f \in g_{\mathbf{R}}^{*}$.

Let $d_{j}(l)=\operatorname{dim}_{\mathbf{C}} G_{j} \cdot p_{j}(l)$ for $l \in \mathfrak{g}^{*}$ and $1 \leq j \leq r$; set $d(l)=\left(d_{1}(l), \ldots, d_{r}(l)\right)$. Because we are dealing with coadjoint orbits, $d_{j}(l)$ is always even; it is also easy to check that $d_{j}(l)-d_{j-1}(l)=0$ or 2 for all $j$. (We define $d_{0}(l)=0$.) Similarly, define $d_{j}(f)=\operatorname{dim}_{\mathbf{R}} H_{j} \cdot p_{j}^{\mathbf{R}}(f)$ for $f \in g_{\mathbf{R}}^{*}$, and set $d(f)=\left(d_{1}(f), \ldots, d_{2 r}(f)\right)$. Let $D=\left\{d \in \mathbf{Z}^{r}: \exists l \in \mathfrak{g}^{*}\right.$ with $\left.d(l)=d\right\}$, and define $D^{\mathbf{R}} \subseteq \mathbf{Z}^{2 r}$ similarly. Define the index contraction map $\alpha: D^{\mathbf{R}} \rightarrow D$ by

$$
\alpha(d)_{j}=\frac{1}{2} d_{2 j}, \quad 1 \leq j \leq r, d \in D^{\mathbf{R}} .
$$

Just as in the proof of Lemma 4.7, it is easy to see that $\alpha$ is a bijection.

Now define the $D$-layers

$$
U_{d}=\{l: d(l)=d\}, \quad d \in D,
$$

and

$$
U_{d}^{\mathbf{R}}=\{f: d(f)=d\}, \quad d \in D^{\mathbf{R}} .
$$


From (5.2), we get

$$
J\left(U_{d}^{\mathbf{R}}\right)=U_{\alpha(d)}, \quad \text { all } d \in D^{\mathbf{R}} .
$$

Now define $S(d)=\left\{j: d_{j}=2+d_{j-1}\right\}, T(d)=\left\{j: d_{j}=d_{j-1}\right\}$ for $d$ in $D$ or $D^{\mathbf{R}}$. It is clear that for $d \in D^{\mathbf{R}}$,

$$
S(\alpha(d))=\left\{j_{1}, \ldots, j_{k}\right\} \Leftrightarrow S(d)=\left\{2 j_{1}-1,2 j_{1}, \ldots, 2 j_{k}-1,2 j_{k}\right\} .
$$

As with the $\mathcal{E}$-layers, we can order the indices in $D^{\mathbf{R}}$ so that $\bigcup\left\{U_{d^{\prime}}^{\mathbf{R}}: d^{\prime} \geq d\right\}$ is Zariski-open in $\mathfrak{g}_{\mathbf{R}}^{*}$ for all $d \in D^{\mathbf{R}}$; the ordering algorithm is explicit, but not unique. There is a similar ordering for $D$, and we can choose the orderings so that $\alpha$ is order-preserving. We should note one dissimilarity between the $D$-layers and the $\mathcal{E}$-layers: the $U_{d}$ are not $G$-invariant in general.

Now let $d$ be the largest index in $D^{\mathbf{R}}$ such that $U_{d}^{\mathbf{R}}$ meets $f^{\prime}+k_{\mathbf{R}}^{\perp}$. The intersection is Zariski-open in $f^{\prime}+k_{\mathbf{R}}^{\perp}$. Since $J\left(f^{\prime}+k_{\mathbf{R}}^{\perp}\right)=\varphi^{\prime}+\mathfrak{k}^{\perp}$ and $J\left(U_{d}^{\mathbf{R}}\right)=U_{\alpha(d)}$, we see that $U_{\alpha(d)}$ meets $\varphi^{\prime}+\mathfrak{k}^{\perp}$ in a Zariski-open set. Hence $\alpha(d)$ is the largest index in $\mathcal{E}$ such that $U_{\alpha(d)}$ meets $\varphi^{\prime}+\mathfrak{k}^{\perp}$. The subspace $E_{1}^{\mathbf{R}}$ is defined as

$$
E_{1}^{\mathbf{R}}=\mathbf{R}-\operatorname{span}\left\{Y_{j}^{*}: j \in T(d)\right\} .
$$

If we define

$$
E_{1}=\mathrm{C}-\operatorname{span}\left\{X_{j}^{*}: j \in T(\alpha(d))\right\},
$$

then $J\left(E_{1}^{\mathbf{R}}\right)=E_{1}$, because

$$
J\left(Y_{2 j-1}^{*}\right)=X_{j}^{*}, \quad J\left(Y_{2 j}^{*}\right)=-i X_{j}^{*}, \quad 1 \leq j \leq r .
$$

Therefore-and this is the key fact we need about $E_{1}^{\mathbf{R}}$ -

$$
J\left(f^{\prime}+E_{1}^{\mathbf{R}}\right)=\varphi^{\prime}+E_{1}
$$

In particular, $J\left(f^{\prime}+E_{1}^{\mathbf{R}}\right)$ is a complex affine subspace in $\mathfrak{g}^{*}$.

We now proceed with the proof of Theorem 1. Let $e \in \mathcal{E}^{\mathbf{R}}$ be the first index such that $U_{e}^{\mathbf{R}}$ meets $f^{\prime}+\mathfrak{k}_{\mathbf{R}}$. From Theorem 3.12, this is also the first index such that $U_{e}^{\mathbf{R}}$ meets $f^{\prime}+E_{1}^{\mathbf{R}}$; we saw above that this intersection is Zariski-open in $f^{\prime}+E_{1}^{\mathbf{R}}$. Let $\varepsilon=\alpha(e)$; let $\pi_{T}, \pi_{S}: \mathfrak{g}^{*}=V_{T(e)} \oplus V_{S(e)} \rightarrow V_{T(e)}, V_{S(e)}$ be the projections, and define

$$
\begin{array}{ll}
S_{1}^{\mathbf{R}}=U_{e}^{\mathbf{R}} \cap\left(f^{\prime}+E_{1}^{\mathbf{R}}\right), & S_{1}=U_{\varepsilon} \cap\left(\varphi^{\prime}+E_{1}\right), \\
\varphi_{\mathbf{R}}=\left.\left(\pi_{T} \circ P_{\mathbf{R}}\right)\right|_{S_{1}^{\mathbf{R}},} & \varphi=\left.\left(\pi_{T} \circ P\right)\right|_{S_{1}}, \\
\Sigma_{1}^{\mathbf{R}}=\varphi_{\mathbf{R}}\left(S_{1}^{\mathbf{R}}\right) \subseteq V_{T(e)}, & \Sigma_{1}=\varphi\left(S_{1}\right) \subseteq V_{T(\varepsilon)} .
\end{array}
$$

Then $S_{1}$ is Zariski-open in the complex variety $\varphi^{i}+E_{1}$. Let $k=\max \left\{\operatorname{rank}_{\mathbf{C}}(d \varphi)_{l}\right.$ : $\left.l \in S_{1}\right\}$, and set

$$
S_{2}=\left\{l \in S_{1}: \operatorname{rank}_{\mathbf{C}}(d \varphi)_{l}=k\right\}, \quad \Sigma_{2}=\varphi\left(S_{2}\right) .
$$

Because $\varphi$ is rational nonsingular, $S_{2}$ is Zariski-open in $\varphi^{\prime}+E_{1}$. Similarly, define $k_{\mathbf{R}}$ to be the maximal rank for $\varphi_{\mathbf{R}}$, and define $S_{2}^{\mathbf{R}} \subseteq S_{1}^{\mathbf{R}}, \Sigma_{2}^{\mathbf{R}}=\varphi_{\mathbf{R}}\left(S_{2}^{\mathbf{R}}\right)$ analogously. Since $J\left(U_{e}^{\mathbf{R}}\right)=U_{\varepsilon}$ and $J\left(V_{T(e)}\right)=V_{T(\varepsilon)}$, we get

$$
J\left(\Sigma_{1}^{\mathbf{R}}\right)=\Sigma_{1}, \quad J\left(S_{1}^{\mathbf{R}}\right)=S_{1} .
$$

Next, define the sets

$$
W_{j}=P\left(S_{j}\right) \subseteq \Sigma_{j} \times V_{S(\varepsilon)}, \quad W_{j}^{\mathbf{R}}=P_{\mathbf{R}}\left(S_{j}^{\mathbf{R}}\right) \subseteq \Sigma_{j}^{\mathbf{R}} \times V_{S(e)} .
$$


These are just parametric versions of $S_{j}, S_{j}^{\mathbf{R}}$.

We may assume for Theorem 1 that we are in the finite multiplicity case, where $\tau_{0}=0$. In [2], we showed that $\tau_{0}=0 \Leftrightarrow k_{\mathbf{R}}=\operatorname{dim}_{\mathbf{R}} E_{1}^{\mathbf{R}} ;$ thus $\varphi_{\mathbf{R}}: S_{2}^{\mathbf{R}} \rightarrow V_{T(e)}$ is a local diffeomorphism, and for $f \in S_{2}^{\mathbf{R}}$ the orbit $G \cdot f$ meets $S_{2}^{\mathbf{R}}$ in isolated points. Moreover, $J^{-1} \circ P \circ J=P_{\mathbf{R}}$; from this it follows that $J^{-1} \circ \varphi \circ J=\varphi_{\mathbf{R}}$, so that

$$
\operatorname{Rank}_{\mathbf{R}}\left((d \varphi)_{J(f)}\right)=\operatorname{Rank}_{\mathbf{R}}\left(\left(d \varphi_{\mathbf{R}}\right)_{f}\right) \text {. }
$$

Since the real rank of a complex analytic map is twice the complex rank, we see that $k_{\mathbf{R}}=2 k$ and that $S_{2}=J\left(S_{2}^{\mathbf{R}}\right)$.

If $A$ is a set in $\mathfrak{g}^{*}$, we write $A^{\#}$ for its Zariski closure. We now establish the following facts:

(i) We have $W_{1}^{\#}=W_{2}^{\#}\left(=W^{\#}\right.$, by definition), $\Sigma_{1}^{\#}=\Sigma_{2}^{\#}\left(=\Sigma^{\#}\right)$, and these sets are irreducible varieties over $\mathbf{C}$;

(ii) $\operatorname{dim}_{\mathbf{C}} W^{\#}=\operatorname{dim}_{\mathbf{C}} \Sigma^{\#}=k$.

Statement (i) is an immediate consequence of Corollary 3.8, since $P: S_{k} \rightarrow W_{k}$ and $\varphi: S_{j} \rightarrow \Sigma_{j}$ are rational nonsingular. The equality of closures holds because $S_{2}$ is Zariski-dense in $S_{1}$ and $P, \varphi$ are Zariski-continuous on $S_{1}$ (Lemma 3.4).

To prove (ii), note first that

$$
W_{2} \text { contains a Zariski-open dense subset } A_{1} \text { of } W_{1}^{\#} \text {. }
$$

[This is an application of Lemma 3.9, with $S=U_{\varepsilon}, T=\Sigma_{\varepsilon} \times V_{S(\varepsilon)}, \varphi=P_{\varepsilon}$, and $E=\varphi^{\prime}+E_{1}$. The lemma then shows that $W_{1}=P_{\varepsilon}\left(S_{1}\right)$ satisfies (5.5). But $\left.P_{\varepsilon}\right|_{S_{1}}$ is birational nonsingular; Corollary 3.7 implies $(5.5)$ for $W_{2}$.] Since $\operatorname{rank}_{\mathbf{C}}(d \varphi)_{l}=$ $k=\operatorname{dim}_{\mathrm{C}} E_{1}$ if $l \in S_{2}$, we get

$$
\operatorname{rank}_{\mathbf{C}}(d P)_{l}=k, \quad \text { all } l \in S_{2},
$$

since $\varphi=\pi_{T(\varepsilon)} \circ P$. Consequently $W_{2}$ is covered by countably many complex manifolds in $V_{T(\varepsilon)} \times V_{S(\varepsilon)}$ having (complex) dimension $k$. Take any complex stratification $S$ of $W^{\#}$ compatible with the set $A_{1} \subseteq W^{\#}$ of (5.5). By definition, $\operatorname{dim}_{\mathrm{C}} W^{\#}=\max \left\{\operatorname{dim}_{\mathrm{C}} S_{i}: S_{i} \in S\right\}$. From (3.2), the dimension of the pieces in $W^{\#} \backslash A_{1}$ is less than the maximum dimension of the pieces in $W^{\#}$. Since $A_{1}$ is covered by $k$-dimensional submanifolds, we have $\operatorname{dim}_{\mathbf{C}} W^{\#}=\operatorname{dim}_{\mathbf{C}} A_{1} \leq k$. On the other hand, $\operatorname{dim}_{\mathrm{C}} W^{\#} \geq \operatorname{dim}_{\mathbf{C}} P\left(S_{2}\right)=k$. Thus $\operatorname{dim}_{\mathrm{C}} W^{\#}=k$. The proof for $\Sigma^{\#}$ is essentially the same.

Note that $\pi_{T}\left(W^{\#}\right)$ is a Zariski dense subset of $\Sigma^{\#}$, since $\pi_{T}\left(W_{j}\right)=\Sigma_{j}$.

We are now ready to apply the main result we need from algebraic geometry. Since it applies only over $\mathbf{C}$, we have had to introduce the complex picture of the action of $G$ on $\mathfrak{g}_{\mathbf{R}}^{*}$. The result may be regarded as an extension of the theorem that all polynomials in $\mathbf{C}[Z]$ of the same degree have the same number of roots (counting multiplicities); more generally, it is an extension of Bezout's theorem (see, e.g., [9]).

(5.7) THEOREM. Let $X, Y$ be irreducible complex varieties in $\mathbf{C}^{m}, \mathbf{C}^{n}$ respectively, of the same complex dimension, and let $p: \mathbf{C}^{m} \rightarrow \mathbf{C}^{n}$ be a polynomial map such that $p(X)$ is dense in $Y$. Then there is a Zariski-open nonempty set $A \subseteq Y$ such that $p$ is a covering map from $B=p^{-1}(A) \cap X$ onto $A$ (in the Euclidean topology). In particular, $A$ and $B$ are topologically connected.

The proof is a modification of the result on pp. 117-120 of [3]; we are indebted to David Rohrlich for valuable help. From Theorem 2.25 of [3], we know that there 
are normal varieties $X_{n}, Y_{n}$, plus morphisms (polynomial maps) $\mu, \nu, p_{n}$, such that

(a) the diagram

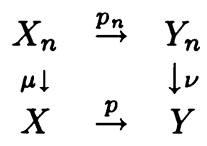

commutes;

(b) there is a proper subvariety $S_{0}$ of $X$ such that $\mu: X_{n} \backslash \mu^{-1}\left(S_{0}\right) \rightarrow X \backslash S_{0}$ is an isomorphism, and there is a proper subvariety $S_{1}$ of $Y$ such that $\nu$ is an isomorphism on $Y_{n} \backslash \nu^{-1}\left(S_{1}\right)$.

The result of [3] cited above says that there is a proper subvariety $S_{2}$ of $Y_{n}$ such that $p_{n}$ is a covering map from $X_{n} \backslash p_{n}^{-1}\left(S_{2}\right)$ to $Y_{n} \backslash S_{2}$. Now let $S$ be the Zariski closure of $S_{1} \cup p\left(S_{0}\right) \cup \nu\left(S_{2}\right)$ in $Y$. Then $Y \backslash S$ is a nonempty Zariski-open set in $Y$, since $\operatorname{dim} S<\operatorname{dim} Y$ (see p. 93 of [3]), and $\left.\nu^{-1}\right|_{Y \backslash S},\left.\mu^{-1}\right|_{X \backslash p^{-1}(S)}$ are isomorphisms. Since $p_{n}$ is a covering map on $\mu^{-1}\left(X_{n} \backslash p^{-1}(S)\right)=p_{n}^{-1}\left(Y_{n} \backslash \nu^{-1}(S)\right)$, we see that $p$ is a covering map on $X \backslash p^{-1}(S)$. This proves the theorem.

Taking $X=W^{\#}, Y=\Sigma^{\#}$, and $p=\pi_{T}$, we get a nonempty Zariski-open subset $A \subseteq \Sigma^{\#}$ such that the covering index

$$
i=i(l)=\operatorname{card}\left\{\pi_{T}^{-1}(l) \cap W^{\#}\right\}=\operatorname{Card}\left\{\left(\{l\} \times V_{S}\right) \cap W^{\#}\right\}
$$

is constant on $A$. This is not quite what we need, since for $f \in \Sigma_{e}^{\mathbf{R}}$ the number $n(f)$ in Theorem 3.12 is given by

$$
\begin{aligned}
n(f) & =\operatorname{Card}\left(\left(E_{1}^{\mathbf{R}}+f^{\prime}\right) \cap G \cdot f\right)=\operatorname{Card}\left(\left(E_{1}+\varphi^{\prime}\right) \cap G \cdot l\right) \\
& =\operatorname{Card}\left(S_{1} \cap P^{-1}\left(\{l\} \times V_{S}\right)\right)=\operatorname{Card}\left(P\left(S_{1}\right) \cap\left(\{l\} \times V_{S}\right)\right) \\
& =\operatorname{Card}\left(W_{1} \cap\left(\{l\} \times V_{S}\right)\right), \quad l=J(f),
\end{aligned}
$$

and thus we need to get from $W^{\#}$ to $W_{1}$. This is not hard. Let $A_{1}$ be a Zariski-open (dense) subset of $W^{\#}$ contained in $W_{1}$, as in (5.5). Then $X=W^{\#} \backslash A_{1}$ has complex dimension less than that of $W^{\#}$, by (3.2). Hence $\pi_{T}(X)$ is a countable union of manifolds in $V_{T}$ having complex dimension $\leq \operatorname{dim}_{\mathrm{C}} X<k$. Thus $A_{0}=A \backslash \pi_{T}(X)$ and $B_{0}=\pi_{T}^{-1}\left(A_{0}\right) \cap W^{\#}$ are sets in $\Sigma^{\#}, W^{\#}$ respectively such that

(i) $B_{0} \subseteq W_{1}$;

(ii) $i(l)=i$ is constant on $A_{0}$, and $\operatorname{dim}\left(\Sigma^{\#} \backslash A_{0}\right)<\operatorname{dim} \Sigma^{\#}$.

From (i) and (5.8), we see that $n(f)=i$ if $f \in J^{-1}\left(A_{0}\right)$. Furthermore, $\operatorname{dim} \Sigma_{2}=$ $\operatorname{dim} W_{2}=\operatorname{dim} W^{\#}=\operatorname{dim} \Sigma^{\#}$. Thus (ii) implies that $J^{-1}\left(A_{0}\right)$ has full measure in $J^{-1}\left(\Sigma^{\#}\right)=\Sigma_{\mathbf{R}}^{\#}$. Theorem 3.12 now gives

$$
\rho \cong \int_{\Sigma_{1}^{0}}^{\oplus} i \pi_{f} d \nu_{1}(f),
$$

which completes the proof of Theorem 1 .

6. This section is devoted to some algebraic preliminaries to the proof of Theorem 2. We write $\mathfrak{g}_{0}, \mathfrak{k}_{0}$ for the (real) Lie algebras of $G_{0}, K_{0}$ respectively, and $\mathfrak{g}, \mathfrak{k}$ for their complexifications; we regard $\mathfrak{k}_{0}, \mathfrak{g}_{0}$ as (real) subalgebras of $\mathfrak{k}, \mathfrak{g}$ respectively. Let $G, K$ be the (complex) Lie groups corresponding to $\mathfrak{g}, \mathfrak{k}$ respectively. As in $\S 4$, we imbed $\mathfrak{g}_{0}^{*}$ in $\mathfrak{g}^{*}$, letting

$$
(I f)(X+i Y)=f(X)+i f(Y), \quad \text { all } f \in \mathfrak{g}_{0}^{*} .
$$


We shall often write $f$ for $I f$. Then $\mathfrak{g}^{*}=\mathfrak{g}_{0}^{*}+i \mathfrak{g}_{0}^{*}$; we regard $\mathfrak{g}_{0}^{*}=\left\{l \in \mathfrak{g}^{*}: l\left(\mathfrak{g}_{0}\right) \subseteq \mathbf{R}\right\}$ as the set of real points in $\mathfrak{g}^{*}$. Fix a (real) Malcev basis $X_{1}, \ldots, X_{n}$ for $\mathfrak{g}_{0}$ and let $\left\{l_{1}, \ldots, l_{n}\right\}$ be the dual basis in $\mathfrak{g}_{0}^{*}$. These are also dual bases (over $\mathbf{C}$ ) for $\mathfrak{g}, \mathfrak{g}^{*}$, and C-span $\left\{l_{j+1}, \ldots, l_{n}\right\}$ is $G$-invariant for all $j$. Define subalgebras and canonical projections,

$$
\begin{aligned}
\mathfrak{g}_{j, 0} & =\mathbf{R}-\operatorname{span}\left\{X_{1}, \ldots, X_{j}\right\}, & & p_{j, 0}: \mathfrak{g}_{0}^{*} \rightarrow \mathfrak{g}_{j, 0}^{*}, \\
\mathfrak{g}_{j} & =\mathbf{C}-\operatorname{span}\left\{X_{1}, \ldots, X_{j}\right\}, & & p_{j}: \mathfrak{g}^{*} \rightarrow \mathfrak{g}_{j}^{*},
\end{aligned}
$$

which commute with the obvious actions of $G_{0}, G$. Write $G_{0} \cdot f, G \cdot l$ for Ad $^{*}$-orbits of $f \in \mathfrak{g}_{0}^{*}, l \in \mathfrak{g}^{*}$, and define dimension indices as in $\S 4$ :

$$
\begin{aligned}
e_{j}(f) & =\operatorname{dim}_{\mathbf{R}} p_{j, 0}\left(G_{0} \cdot f\right), & e(f) & =\left(e_{1}(f), \ldots, e_{n}(f)\right)\left(f \in \mathfrak{g}_{0}^{*}\right), \\
\varepsilon_{j}(l) & =\operatorname{dim}_{\mathbf{C}} p_{j}(G \cdot l), & \varepsilon(l) & =\left(\varepsilon_{1}(l), \ldots, \varepsilon_{n}(l)\right)\left(l \in \mathfrak{g}^{*}\right),
\end{aligned}
$$

index sets $\mathcal{E}_{0}=\left\{e \in \mathbf{Z}^{n}: \exists f \in \mathfrak{g}_{0}^{*}\right.$ with $\left.e=e(f)\right\}, \mathcal{E}=\left\{\varepsilon \in \mathbf{Z}^{n}: \exists l \in \mathfrak{g}^{*}\right.$ with $\varepsilon=\varepsilon(l)\}$, and layers

$$
U_{e}^{0}=\left\{f \in \mathfrak{g}_{0}^{*}: e(f)=e\right\}, \quad e \in \mathcal{E}_{0}, \quad U_{\varepsilon}=\left\{l \in \mathfrak{g}^{*}: \varepsilon(l)=\varepsilon\right\}, \quad \varepsilon \in \mathcal{E} .
$$

The parametrization of Theorem 2.1 applies both to the action of $G_{0}$ on $\mathfrak{g}_{0}^{*}$ and to that of $G$ on $\mathfrak{g}^{*}$. For each layer, we have cross-sectioning vector subspaces $V_{T(e)}^{0}, V_{T(\varepsilon)}$, and sets of orbit representatives $\Sigma_{e}^{0}, \Sigma_{\varepsilon}$. The connections between these objects are given by

(6.1) PROPOSITION. In the preceding situation, we have

(i) $f \in \mathfrak{g}_{0}^{*} \Rightarrow e(f)=\varepsilon(I f)$. Thus $\mathcal{E}^{0} \subseteq \mathcal{E}$ in $\mathbf{Z}^{n}$.

(ii) For any $e \in \mathcal{E}^{0}$,

$$
U_{e}^{0}=U_{e} \cap \mathfrak{g}_{0}^{*}, \quad \Sigma_{e}^{0}=\Sigma_{e} \cap \mathfrak{g}_{0}^{*} .
$$

(iii) One can choose orderings of $\mathcal{E}^{0}, \mathcal{E}$ as in Theorem 2.1(ii) so that the inclusion $\mathcal{E}^{0} \subseteq \mathcal{E}$ is order-preserving.

(iv) For $e \in \mathcal{E}^{0}$, form the birational parametrizing maps

$$
P_{e}^{0}: U_{e}^{0} \rightarrow \Sigma_{e}^{0} \times V_{S(e)}^{0}, \quad P_{e}: U_{e} \rightarrow \Sigma_{e} \times V_{S(e)}
$$

of Theorem 2.1(iv). Then

$$
V_{S(e)}=V_{S(e)}^{0} \otimes_{\mathbf{R}} \mathbf{C}=V_{S(e)}^{0}+i V_{S(e)}^{0}, \quad V_{T(e)}=V_{T(e)}^{0}+i V_{T(e)}^{0}
$$

and $P_{0}^{e}$ is the restriction of $P_{e}$.

ProOF. For $l \in \mathfrak{g}^{*}$, let $\mathfrak{h}_{j}=\mathfrak{h}_{j}(l)=\left\{X \in \mathfrak{g}:\left(\operatorname{ad}^{*} X\right) p_{j}(l)=0\right\}$, and set $\mathfrak{h}_{0}=\mathfrak{g}$; for $f \in \mathfrak{g}_{0}^{*}$, set $\mathfrak{h}_{j, 0}=\mathfrak{h}_{j, 0}(f)=\left\{X \in \mathfrak{g}_{0}: \operatorname{ad}^{*}(X) p_{j, 0}(f)=0\right\}, \mathfrak{h}_{0,0}=\mathfrak{g}_{0}$. It follows from the proof of Theorem 2.1 that for $f \in \mathfrak{g}_{0}^{*}$,

$$
e_{j}(f)=\operatorname{codim}_{\mathbf{R}} \mathfrak{h}_{j, 0}(f)
$$

while for $l \in \mathfrak{g}^{*}$,

$$
\varepsilon_{j}(l)=\operatorname{codim}_{\mathbf{C}} \mathfrak{h}_{j}(l) .
$$

If $f \in \mathfrak{g}_{0}^{*} \subseteq \mathfrak{g}^{*}$, it is easy to check that $\mathfrak{h}_{j}(f)=\mathfrak{h}_{j, 0}(f)+i \mathfrak{h}_{j, 0}(f)$. This proves (i), and the first part of (ii) follows immediately. The ordering algorithm (as in $\S 2$ of [2]) implies (iii) easily. For $\varepsilon \in \mathcal{E}$, we define $S(\varepsilon)=\left\{j: \varepsilon_{j}>\varepsilon_{j-1}\right\}$ and 
$T(\varepsilon)=\left\{j: \varepsilon_{j}=\varepsilon_{j-1}\right\}$; if $e \in \mathcal{E}^{0} \subseteq \mathcal{E}$, these sets are the same whether we regard $e$ as in $\mathcal{E}^{0}$ or $\mathcal{E}$. The spaces $V_{S(e)}, V_{T(e)} \subseteq \mathfrak{g}^{*}$ are the complex spans of the $l_{i}$ with the appropriate indices, and $V_{S(e)}^{0}, V_{T(e)}^{0}$ are the corresponding real spaces. Obviously we have

$$
V_{S(e)}^{0}=V_{S(e)} \cap \mathfrak{g}_{0}^{*}, \quad V_{T(e)}^{0}=V_{T(e)} \cap \mathfrak{g}_{0}^{*} \quad \text { for } e \in \mathcal{E}^{0} ;
$$

since $\Sigma_{e}=V_{S(e)} \cap U_{e}$ and $\Sigma_{e}^{0}=V_{S(e)}^{0} \cap U_{e}^{0}$, the second part of (ii) holds.

We have now proved everything but the last claim in (iv). To construct the inverse map $Q_{e}=P_{e}^{-1}: \Sigma_{e} \times V_{S(e)} \rightarrow U_{e}$ for $e \in \mathcal{E}$, one chooses rationally varying elements $Y_{j}(l) \in \mathfrak{h}_{j-1}(l) \backslash \mathfrak{h}_{j}(l)$ such that $p_{j}\left(\operatorname{ad}^{*} Y_{j}(l)\right)=l_{j}$ for each index $j \in S(e)$. When $l \in \mathfrak{g}_{0}^{*}$, the same construction, verbatim, yields $Q_{e}^{0}=\left(P_{e}^{0}\right)^{-1}$. Since we can choose $Y_{j}(l) \in \mathfrak{g}_{0}$ when $l \in \mathfrak{g}_{0}^{*}$, the polynomials $P_{e}, Q_{e}$ have real coefficients when $e \in \mathcal{E}_{0}$, and the constructions show that $P_{e}^{0}, Q_{e}^{0}$ are the restrictions of $P_{e}, Q_{e}$ respectively. (Notice that if $e \in \mathcal{E}$, then $U_{e} \subseteq \mathfrak{g}^{*}$ meets $\mathfrak{g}_{0}^{*}$ iff $e \in \mathcal{E}_{0}$, by (i).)

REMARK. We do not know whether the inclusion $\mathcal{E}^{0} \subseteq \mathcal{E}$ is proper in general.

(6.2) Proposition. If $f \in \mathfrak{g}_{0}^{*}$, then $G \cdot f \cap \mathfrak{g}_{0}^{*}=G_{0} \cdot f$.

PROOF. We use the geometric description of the parametrizing maps, cited in Theorem 2.1(vi). Choose a Malcev basis for $\mathfrak{g}_{0}$ and induce layerings of $\mathfrak{g}_{0}^{*}, \mathfrak{g}^{*}$ as above; let $e$ be the index with $f_{0} \in U_{e}^{0}=U_{e} \cap \mathfrak{g}_{0}^{*}$, and let $\pi_{S}, \pi_{T}$ be the projections for $\mathfrak{g}^{*}=V_{S(e)} \oplus V_{T(e)}$. $\pi_{S}$ is a surjective polynomial diffeomorphism of $G \cdot f$ onto $V_{S(e)}$. Similarly, if $\pi_{S}^{0}, \pi_{T}^{0}$ are the projections for $\mathfrak{g}_{0}^{*}=V_{S(e)}^{0} \oplus V_{T(e)}^{0}$, then $\pi_{S}^{0}$ is a surjective polynomial diffeomorphism of $G_{0} \cdot f$ onto $V_{S(e)}^{0}=V_{S(e)} \cap \mathfrak{g}_{0}^{*}$. In view of Proposition 6.1(iv), $\pi_{S}$ maps $G_{0} \cdot f$ (which is in $\mathfrak{g}_{0}^{*}$ ) onto $\pi_{S}^{0}\left(G_{0} \cdot f\right)=V_{S}^{0}$. Since $\pi_{S}$ maps any real point in $G \cdot f$ onto $V_{S}^{0}$, we see that $G_{0} \cdot f$ accounts for all the real points in $G \cdot f$, as required.

7. We now prove Theorem 2 . We distinguish various Zariski closures as follows: if $S \subseteq \mathfrak{g}^{*}$, its (complex) Zariski hull is $S^{\mathbf{C}}$, while if $S \subseteq \mathfrak{g}_{0}^{*}$, its (real) Zariski hull in $\mathfrak{g}_{0}^{*}$ is $S^{\#}$. Pick a real Malcev basis for $\mathfrak{g}_{0}^{*}$ and partition $\mathfrak{g}_{0}^{*}, \mathfrak{g}^{*}$ into layers as in Theorem 2.1 and Proposition 6.1. As in Theorem 1, we may assume that $\chi \in K^{\wedge}$ is 1-dimensional. Thus $\chi=e^{2 \pi i f}$ for some $f \in \mathfrak{k}_{0}^{*}$. Then $P^{-1}\left(O_{\chi}\right)=$ $f^{\prime}+\mathfrak{k}_{0}^{\perp}$, where $f^{\prime} \in \mathfrak{g}_{0}^{*}$ is any extension of $f$. Let $e \in \mathcal{E}^{0}$ be the first index such that $U_{e}^{0}$ meets $f^{\prime}+\mathfrak{k}_{0}^{\perp}$. If we split $\mathfrak{k}_{0}^{\perp}=E_{1}^{0} \oplus E_{2}^{0}$ as in Theorem 3.12, then $U_{e}^{0}$ is also the first layer to meet $f^{\prime}+E_{1}^{0}$; their intersection is Zariski-open in $f^{\prime}+E_{1}^{0}$, by Theorem 2.1(ii).

Now define

$$
\begin{aligned}
S_{1}^{0} & =U_{e}^{0} \cap\left(f^{\prime}+E_{1}^{0}\right), \\
\varphi_{0} & =\left.\pi_{T}^{0} \circ P_{e}^{0}\right|_{S_{1}^{0}} \quad\left(\text { thus } \varphi_{0}: S_{1}^{0} \rightarrow V_{T}^{0}\right), \\
\Sigma_{1}^{0} & =\operatorname{range} \varphi_{0}=\varphi_{0}\left(S_{1}^{0}\right) \subseteq \Sigma_{e}^{0}, \\
k & =\max \left\{\operatorname{rank}_{\mathbf{R}}\left(d \varphi_{0}\right)_{f}: f \in S_{1}^{0}\right\}, \\
S_{2}^{0} & =\left\{f \in S_{1}^{0}: \operatorname{rank}_{\mathbf{R}}\left(d \varphi_{0}\right)_{f}=k\right\}, \\
\Sigma_{2}^{0} & =\varphi_{0}\left(S_{2}^{0}\right) \subseteq \Sigma_{1}^{0} .
\end{aligned}
$$

Then $S_{1}^{0}$ and $S_{2}^{0}$ are Zariski-open in $f^{\prime}+E_{1}^{0}$ (recall that $\varphi$ is rational). In $\S 5$ of [2], we showed that the condition $\tau_{0}=0$ is equivalent to $k=\operatorname{dim}_{\mathbf{R}} E_{1}^{0}$, or to

$$
\varphi_{0}: S_{2}^{0} \rightarrow \Sigma_{2}^{0} \text { is a local diffeomorphism. }
$$


For the rest of the proof, we shall assume (as we may) that $\tau_{0}=0$.

Let $\varepsilon \in \mathcal{E}$ be the first index such tht $U_{\varepsilon}$ meets $f^{\prime}+E_{1}$, where $E_{1}=E_{1}^{0}+i E_{1}^{0}$. Since $U_{\varepsilon} \cap\left(f^{\prime}+E_{1}\right)$ is Zariski-open in $f^{\prime}+E_{1}$ and $f^{\prime}+E_{1}^{0}$ is Zariski-dense in $f^{\prime}+E_{1}$, we see that $U_{\varepsilon}$ meets $f^{\prime}+E_{1}^{0}$. Hence $\varepsilon \in \mathcal{E}^{0}$, and it is now clear that $\varepsilon=e$ (the "first index" for $f^{\prime}+E_{1}^{0}$ ).

Now define the parametric versions of the sets $S_{j}^{0}$ :

$$
W_{j}^{0}=P_{e}^{0}\left(S_{j}^{0}\right), \quad j=1,2,
$$

where $P_{e}^{0}: U_{e}^{0} \rightarrow \Sigma_{e}^{0} \times V_{S(e)}^{0}$ is the birational nonsingular parametrizing map for the layer $U_{e}^{0}$. Since $\varphi_{0}=\left.\pi_{T}^{0} \circ P_{e}^{0}\right|_{S_{1}^{0}}$, we have

$$
\pi_{T}^{0}\left(W_{j}^{0}\right)=\Sigma_{j}^{0}, \quad j=1,2 .
$$

From Corollary 3.8, we know that

$\left(\Sigma_{1}^{0}\right)^{\#}=\left(\Sigma_{2}^{0}\right)^{\#}\left(=\Sigma^{\#}\right.$, by definition $)$ is irreducible in $V_{T(e)}^{0} ;$

$\left(W_{1}^{0}\right)^{\#}=\left(W_{2}^{0}\right)^{\#}\left(=W^{\#}\right)$ is irreducible in $\mathfrak{g}_{0}^{*}$.

From Theorem 3.12, we have

$$
\rho \cong \int_{\Sigma_{1}^{0}}^{\oplus} n(l) \pi_{l} d \nu_{1}(l), \quad n(l)=\operatorname{card}\left(G_{0} \cdot l \cap\left(f^{\prime}+E_{1}^{0}\right)\right),
$$

where $\left[\nu_{1}\right]$ is the canonical measure class on the semialgebraic set $\Sigma_{1}^{0}$. Moreover, $W_{1}^{0}$ contains a Zariski-open, dense subset of $W^{\#}$, by Lemma 3.9, with $S=U_{e}^{0}, T=$ $\Sigma_{e}^{0} \times V_{S(e)}^{0}, \varphi(S \cap E)=W_{1}^{0}$. Hence,

$$
\operatorname{dim}_{\mathbf{R}}\left(W^{\#} \backslash W_{1}^{0}\right)<\operatorname{dim}_{\mathbf{R}} W^{\#} .
$$

Since $W_{1}^{0}$ and $S_{1}^{0}$ are diffeomorphic and $S_{1}^{0}$ is Zariski-open in the $k$-dimensional space $f^{\prime}+E_{1}^{0}$, we have $\operatorname{dim}_{\mathbf{R}} W_{1}^{0}=k$. Thus $\operatorname{dim}_{\mathbf{R}} W^{\#}=k$; Proposition 3.3 says that

$$
k=\operatorname{dim}_{\mathbf{R}} W^{\#}=\operatorname{dim}_{\mathbf{C}}\left(W^{\#}\right)^{\mathbf{C}} .
$$

Because $\varphi_{0}: S_{1}^{0} \rightarrow \Sigma_{1}^{0}$ is a local diffeomorphism, we can reason similarly to show that

$$
k=\operatorname{dim}_{\mathbf{R}} \Sigma^{\#}=\operatorname{dim}_{\mathbf{C}}\left(\Sigma^{\#}\right)^{\mathbf{C}} .
$$

For convenience, we write $W^{\mathbf{C}}=\left(W^{\#}\right)^{\mathbf{C}}, \Sigma^{\mathbf{C}}=\left(\Sigma^{\#}\right)^{\mathbf{C}}$. Note that $W^{\mathbf{C}}, \Sigma^{\mathbf{C}}$ are irreducible because $f^{\prime}+E_{1}^{0}$ is (use Lemma 3.1 and Corollary 3.8).

Now consider $\pi_{T}: W^{\mathbf{C}} \rightarrow \Sigma^{\mathbf{C}}$. Theorem 5.7 applies; thus there are Zariski-open, nonempty sets $A \subseteq \Sigma^{\mathbf{C}}, B=\pi_{T}^{-1}(A) \cap W^{\mathbf{C}}$ such that $\pi_{T}: B \rightarrow A$ is a topological covering map. The set $A$ is topologically connected, and there is an integer $i$ such that

$$
i=i(l)=\operatorname{card}\left(\pi_{T}^{-1}(l) \cap W^{\mathbf{C}}\right), \quad \text { all } l \in A .
$$

Let $A_{0}=A \cap \Sigma^{\#}$. Then $A_{0}$ is nonempty (since $\Sigma^{\#}$ is Zariski-dense in $\Sigma^{\mathbf{C}}$ ); it is easy to see that $A_{0}$ is Zariski-open in $\Sigma^{\#}$. Proposition 3.3 gives

$$
\operatorname{dim}_{\mathbf{R}}\left(\Sigma^{\#} \backslash A_{0}\right)<\operatorname{dim}_{\mathbf{R}} \Sigma^{\#}=k .
$$

Furthermore, $A_{0}$ must meet $\Sigma_{1}^{0}$ because $\Sigma^{\#}=\left(\Sigma_{1}^{0}\right)^{\#}$; letting $A_{1}=A_{0} \cap \Sigma_{1}^{0}$, we have $\Sigma_{1}^{0} \backslash A_{1} \subseteq \Sigma^{\#} \backslash A_{0}$, so that

$$
\operatorname{dim}_{\mathbf{R}}\left(\Sigma_{1}^{0} \backslash A_{1}\right)<k .
$$

Hence $\left.\nu_{1}\right|_{A_{1}}=\nu_{1}$ in the decomposition (7.2). 
We now relate $i(l)$ to $n(l)$ for $l \in A_{1}$. Take bases in $V_{T(e)}^{0}, V_{S(e)}^{0}$ to get coordinates $(u, x)$ in $V_{T(e)}^{0} \times V_{S(e)}^{0}$; these bases are also bases (over $\left.\mathbf{C}\right)$ for $V_{T(e)}, V_{S(e)}$, and they thus give complex coordinates $(w, z)$ in $V_{T(e)} \times V_{S(e)}$. Since $W^{\mathbf{C}}$ is the Zariskiclosure of a set of real points, there are polynomials $p_{1}, \ldots, p_{N} \in \mathbf{R}\left[X_{1}, \ldots, X_{n}\right]$ such that

$$
W^{\mathbf{C}}=\bigcap_{j=1}^{N}\left\{(w, z) \in V_{T(e)} \times V_{S(e)}=\mathfrak{g}^{*}: p_{j}(w, z)=0\right\}
$$

The map $\pi_{T}$ simply maps $(w, z)$ to $(w, 0)$. It is clear that $(w, z) \in W^{\mathbf{C}} \Leftrightarrow(\bar{w}, \bar{z}) \in$ $W^{\mathbf{C}}$ (where bars denote complex conjugates). In particular, if $u$ is real, then the elements $(u, z) \in W^{\mathrm{C}}$ with $z \notin V_{S(e)}^{0}$ come in conjugate pairs. So if

$$
j(l)=\operatorname{Card}\left\{\pi_{T}^{-1}(l) \cap W^{\#}\right\}, \quad l \in A_{1},
$$

then $j(l) \equiv i(l) \equiv i \bmod 2$.

To conclude the proof of Theorem 2, it suffices to show that $j(l)=n(l)$ (where $n(l)$ is as in (7.2)) for $\nu_{1}$-almost all $l \in A_{1}$, since in that case we will have $n(l) \equiv$ $i \bmod 2$ a.e. The proof of this is like the proof at the end of $\S 5$. Let $X=W^{\#} \backslash W_{1}^{0}$. Since $W^{\#}$ is irreducible and $W_{1}^{0}$ is Zariski-open in $W^{\#}, \operatorname{dim}_{\mathbf{R}} X<k=\operatorname{dim}_{\mathbf{R}} W^{\#}$. Hence $\operatorname{dim}_{\mathbf{R}} \pi_{T}^{0}(X)<k=\operatorname{dim} \Sigma_{1}^{0}=\operatorname{dim} \Sigma^{\#}$, or $\nu_{1}\left(\pi_{T}^{0}(X)\right)=0$. Off $\pi_{T}^{0}(X)$, however, $j(l)=n(l)$ because their definitions are essentially the same. This completes the proof.

REMARK. We have actually proved a bit more than Theorem 2: we have shown that except for a set of lower dimension in $\Sigma^{\#}, n(l)$ has constant parity. There are cases in which $\Sigma^{\#}$ is appreciably larger than $\Sigma_{0}^{1}$ (see the next section); when these cases arise, the number $n(l)$ must be (a.e.) even, since $n(l)$ is then 0 on a large subset of $\Sigma^{\#}$.

8. Here we give two examples, related to each other and to two examples in [2].

Let $\mathfrak{g}$ be the 5-dimensional complex Lie algebra spanned by $X_{1}, X_{2}, X_{3}, X_{4}, X_{5}$, with nonzero brackets $\left[X_{5}, X_{4}\right]=X_{3},\left[X_{5}, X_{3}\right]=X_{2},\left[X_{5}, X_{2}\right]=X_{1}$. We denote by $\mathfrak{g}_{0}$ the real algebra with the same brackets.

The given basis is a Malcev basis for $\mathfrak{g}$, with dual basis $l_{1}, \ldots, l_{5}$ in $\mathfrak{g}^{*}$. The elements of $\mathcal{E}$ and the corresponding layerings and decompositions are:

(a) $e_{1}=(0,1,1,1,2) ; U_{e_{1}}=\left\{l=\sum_{j=1}^{5} \alpha_{j} l_{j}: \alpha_{1} \neq 0\right\}, V_{S}=\mathbf{C}-\operatorname{span}\left\{l_{2}, l_{5}\right\}, V_{T}$ $=\mathrm{C}-\operatorname{span}\left\{l_{1}, l_{3}, l_{4}\right\}, \Sigma_{e_{1}}=\left\{\alpha_{1} l_{1}+\alpha_{3} l_{3}+\alpha_{4} l_{4}: \alpha_{1} \neq 0\right\}$, and the orbit map $Q=Q_{e_{1}}$ is given by

$$
\begin{aligned}
& Q\left(\alpha_{1} l_{1}+\alpha_{3} l_{3}+\alpha_{4} l_{4} ; z l_{2}+w l_{5}\right) \\
& \quad=\alpha_{1} l_{1}+z l_{2}+\left(\alpha_{3}+\frac{z^{2}}{2 \alpha_{1}}\right) l_{3}+\left(\alpha_{4}+\frac{\alpha_{3} z}{\alpha_{1}}+\frac{z^{3}}{6 \alpha_{1}^{2}}\right) l_{4}+w l_{5} .
\end{aligned}
$$

(b) $e_{2}=(0,0,1,1,2) ; U_{e_{2}}=\left\{l=\sum_{j=2}^{5} \alpha_{j} l_{j}: \alpha_{2} \neq 0\right\}, V_{S}=\mathrm{C}-\operatorname{span}\left\{l_{3}, l_{5}\right\}, V_{T}$ $=\mathbf{C}-\operatorname{span}\left\{l_{1}, l_{2}, l_{4}\right\}, \Sigma_{e_{2}}=\left\{\alpha_{2} l_{2}+\alpha_{4} l_{4}: \alpha_{2} \neq 0\right\}$, and $Q=Q_{e_{2}}$ is given by

$$
Q\left(\alpha_{2} l_{2}+\alpha_{4} l_{4} ; z l_{3}+w l_{5}\right)=\alpha_{2} l_{2}+z l_{3}+\left(\frac{z^{2}}{2 \alpha_{2}}+\alpha_{4}\right) l_{4}+w l_{5}
$$


(c) $e_{3}=(0,0,0,1,2) ; U_{e_{2}}=\left\{l=\sum_{j=3}^{5} \alpha_{j} l_{j}: \alpha_{3} \neq 0\right\}, V_{S}=\mathrm{C}-\operatorname{span}\left\{l_{4}, l_{5}\right\}, V_{T}=$ C-span $\left\{l_{1}, l_{2}, l_{3}\right\}, \Sigma_{e_{3}}=\left\{\alpha_{3} l_{3}: \alpha_{3} \neq 0\right\}$, and $Q=Q_{e_{3}}$ is given by

$$
Q\left(\alpha_{3} l_{3} ; z l_{4}+w l_{5}\right)=\alpha_{3} l_{3}+z l_{4}+w l_{5} .
$$

(d) $e_{4}=(0,0,0,0,0) ; U_{e_{4}}=\left\{l=\sum_{j=4}^{5} \alpha_{j} l_{j}\right\}, V_{S}=\{0\}, V_{T}=\mathfrak{g}^{*}, \Sigma_{e_{4}}=U_{e_{4}}$, and $Q: \Sigma_{e_{4}} \times\{0\} \rightarrow \Sigma_{e_{4}}$ is the obvious map.

We identify $\operatorname{Ad}^{*}(G)$-orbits in $\mathfrak{g}^{*}$ with elements of $G^{\wedge}$ (where $G$ is the simply connected group corresponding to $\mathfrak{g}$ ) via the Kirillov correspondence and the $J$ map. The representation corresponding to $l$ in some $\Sigma_{e_{j}}$ will be denoted by $\pi_{l}$.

1. Let $\mathfrak{k}_{1}=\mathrm{C}$-span $\left(X_{4}\right)$, and let $\sigma=\chi_{\alpha}$ correspond to the functional $\alpha\left(X_{4}\right)=\alpha$. Then $\alpha$ is the restriction to $\mathfrak{k}_{1}$ of $\alpha l_{4}$, and

$$
\alpha l_{4}+\mathfrak{k}_{1}^{\perp}=\left\{\alpha_{1} l_{1}+\alpha_{2} l_{2}+\alpha_{3} l_{3}+\alpha l_{4}+\alpha_{5} l_{5}: \alpha_{1}, \alpha_{2}, \alpha_{3}, \alpha_{5} \in \mathbf{C}\right\} .
$$

Hence $\alpha l_{4}+\mathfrak{k}_{1}^{\perp}$ meets $U_{e_{1}}$. If $l=\alpha_{1} l_{1}+\alpha_{3} l_{3}+\alpha_{4} l_{4} \in \Sigma_{e_{1}}$, then

$$
\begin{array}{r}
G \cdot \ln \left(\alpha l_{4}+\mathfrak{k}_{1}^{\perp}\right)=\left\{l+z l_{2}+\frac{z^{2}}{2 \alpha_{1}} l_{3}+\left(\frac{\alpha_{3} z}{\alpha_{1}}+\frac{z^{3}}{6 \alpha_{1}^{2}}\right) l_{4}+w l_{5}:\right. \\
\left.w, z \in \mathbf{C}, \frac{z^{3}}{6 \alpha_{1}^{2}}+\frac{a_{3} z}{\alpha_{1}}+\alpha_{4}=\alpha\right\} .
\end{array}
$$

This set consists (generically) of three complex lines, one for each of the roots of the cubic polynomial. Each line is an $\operatorname{Ad}^{*}\left(K_{1}\right)$-orbit. Thus

$$
\operatorname{Ind}\left(K_{1} \uparrow G ; \chi_{\alpha}\right) \cong \int_{\Sigma_{e_{1}}}^{\oplus} 3 \pi_{l} d \nu(l)
$$

where $K_{1}=\exp \mathfrak{k}_{1}$ and $\nu(l)$ is 3-dimensional Lebesgue measure.

2. Let $\mathfrak{k}_{2}=\mathrm{C}-\operatorname{span}\left(X_{1}, X_{4}\right)$ and let $\sigma=\chi_{\beta}$ correspond to the functional $\beta\left(X_{4}\right)=$ $\beta, \beta\left(X_{1}\right)=0$. Then $\beta$ is the restriction to $\mathfrak{k}_{2}$ of $\beta l_{4}$, and

$$
\beta l_{4}+\mathfrak{k}_{2}^{\perp}=\left\{\alpha_{2} l_{2}+\alpha_{3} l_{3}+\beta l_{4}+\alpha_{5} l_{5}: \alpha_{2}, \alpha_{3}, \alpha_{5} \in \mathbf{C}\right\} .
$$

Hence $\beta l_{4}+\mathfrak{k}_{2}^{\perp}$ misses $U_{e_{1}}$, but meets $U_{e_{2}}$. For $l=\alpha_{2} l_{2}+\alpha_{4} l_{4} \in \Sigma_{e_{2}}$,

$$
O_{l} \cap\left(\beta l_{4}+\mathfrak{k}_{2}^{\perp}\right)=\left\{l+z l_{3}+\frac{z^{2}}{2 \alpha_{2}} l_{4}+w l_{5}: z, w \in \mathbf{C}, \frac{z^{2}}{2 \alpha_{2}}+\alpha_{4}=\beta\right\}
$$

a union (generically) of two complex lines corresponding to the roots of the polynomial $z^{2} / 2 \alpha_{2}+\alpha_{4}=\beta$. Each line is an $\operatorname{Ad}^{*}\left(K_{2}\right)$-orbit. Hence

$$
\operatorname{Ind}\left(K_{2} \uparrow G ; \chi_{\beta}\right) \cong \int_{\Sigma_{e_{2}}}^{\oplus} 2 \pi_{l} d \nu(l)
$$

where $\nu(l)$ is 2-dimensional Lebesgue measure.

These examples correspond to Examples 4 and 2 of [2], where we performed calculations equivalent to the above ones for $\mathfrak{g}_{0}$ and $\mathfrak{k}_{1,0}, \mathfrak{k}_{2,0}$ (these latter are the real forms of the $\mathfrak{k}_{j}$ ). For $\mathfrak{k}_{2,0}$, the multiplicity was always 2 , but the integral was taken over $\left\{l=\alpha_{2} l_{2}+\alpha_{4} l_{4} \in \Sigma_{e_{2}}^{0}: \alpha_{2}, \alpha_{4} \in \mathbf{R}\right.$ and $\left.\alpha_{2}\left(\alpha_{4}-\beta\right)>0\right\}$. The intersection of the Zariski closure of this set with $\Sigma_{e_{2}}^{0}$ is all of $\Sigma_{e_{2}}^{0}$; as the note at the end of $\S 7$ states, the parity of the multiplicity is constant on (almost) all of $\Sigma_{e_{2}}^{0}$. 


\section{REFERENCES}

1. L. Corwin and F. P. Greenleaf, Unitary representations of nilpotent Lie groups and applications, Vol. 1, Cambridge Univ. Press, Cambridge, to appear in 1988.

2. L. Corwin, F. P. Greenleaf and G. Grelaud, Direct integral decompositions and multiplicities for induced representations of nilpotent Lie groups, Trans. Amer. Math. Soc. 304 (1987), 537-547.

3. S. Iitaka, Algebraic geometry, Graduate Texts in Math., no. 76, Springer-Verlag, Berlin and New York, 1982.

4. D. Mumford, Algebraic geometry I: Complex projective varieties, Grundlehren Math. Wiss., no. 221, Springer-Verlag, Berlin and New York, 1976.

5. L. Pukanszky, Lecons sur les représentations des groupes, Dunod, Paris, 1967.

6. __ Unitary representations of solvable Lie groups, Ann. Sci. École Norm. Sup. 4 (1971), $457-608$.

7. I. Shafarevich, Introduction to algebraic geometry, Grundlehren Math. Wiss., no. 213, SpringerVerlag, New York, 1975.

8. H. Sussmann, Analytic stratifications and subanalytic sets (in preparation).

9. B. Van der Waerden, Modern algebra, Vol. 2, 2nd ed., Ungar, New York, 1949.

10. A. Weil, Foundations of algebraic geometry, Amer. Math. Soc. Colloq. Publ., vol. 29, Amer. Math. Soc., Providence, R.I., 1962.

Department of Mathematics, Rutgers University, New Brunswick, NeW JerSEY 08903

Department of Mathematics, NYU/Courant Institute, New York, New York 10012 